Received 29 April 2019; accepted 1 July 2019.

Available online 1 October 2019

\title{
الهندسة الكسورية كنمط بنائي للتصميم في العمارة \\ Fractal Geometry as a Structural Pattern for Design in Architecture
}

\author{
أ.م.د/ منى محمد حسني عجور \\ أستاذ مساعد بقسم الهندسة المعمارية

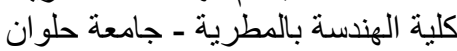 \\ Mona.mhosni@gmail.com
}

الملخص

أثرت الطفرة الرقمية وتطبيقات الحاسب الآلي المستخدمة في العمارة تحديداً التطبيقات و البرامج ثلاثية الأبعاد في فكر وطرق التصديم

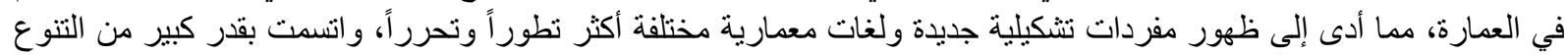

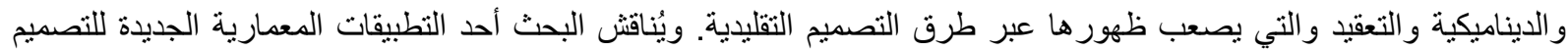

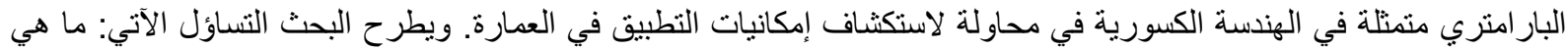

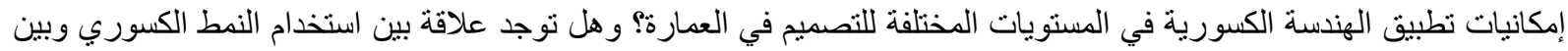
تحقيق وظائف الفر اغات و الحلول البيئية و غير ها من جو انب التصميم واعتبار اته؟

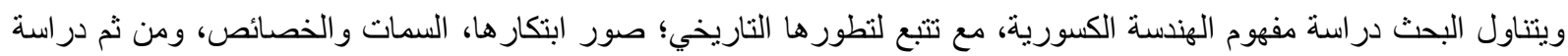

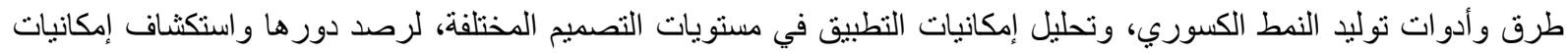

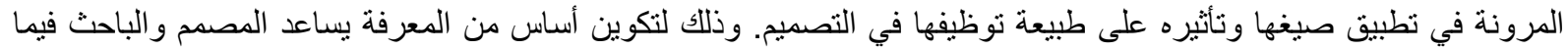
يخص هذه اللغة من البرمجة وتحديد إمكانيات تفعيل هذا النمط في التصميم ومحدداته لاستحداث نتاجات تصميمية مبتكرة بما يلائم المحيط، وبيان الدروس المستفادة و العائد على المجتمع.

ويُختتم البحث بالنتائج والتوصيات، والتي من أهمها أن الهندسة الكسورية من أفضل الخيارات لصياغة التئة الأفكار التصميمية المبتكرة

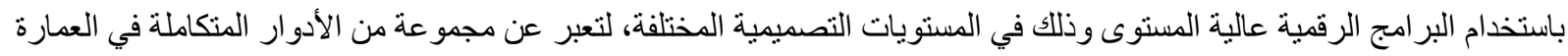

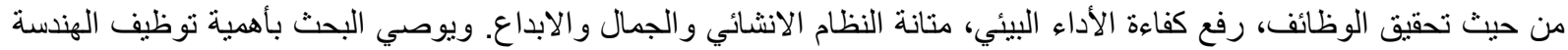

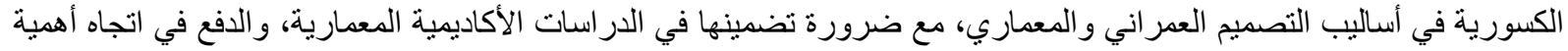

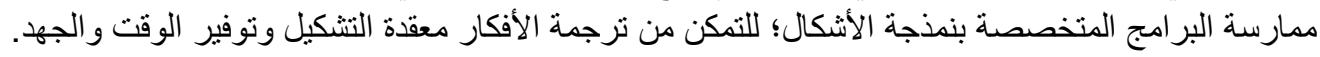

الكلمات المفتاحية:

كسوري Fractal، الهندسة الكسورية، التصميم الكسوري Fractal Design، التشابه الذاتي Self-Similarity، الخوارزميات.

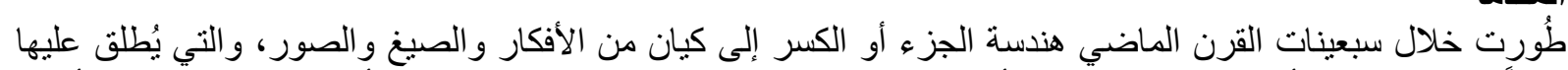

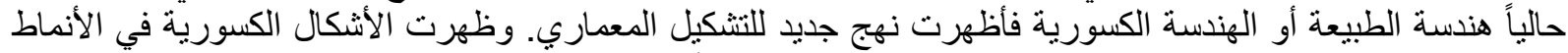

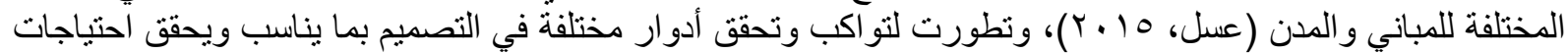

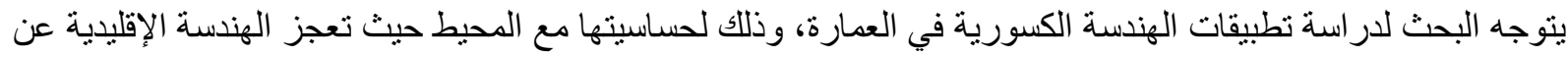

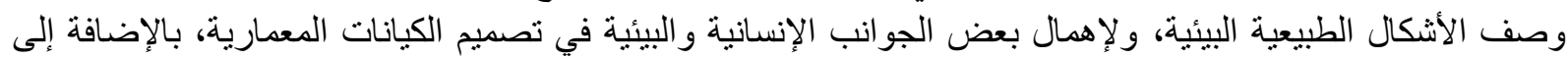

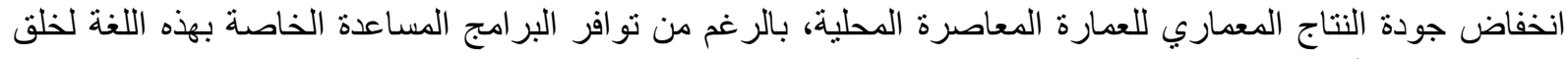

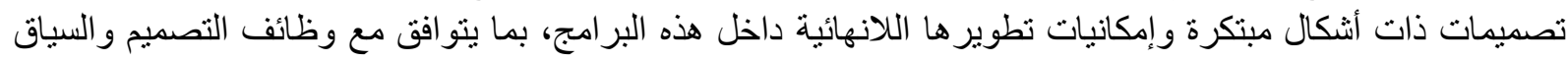

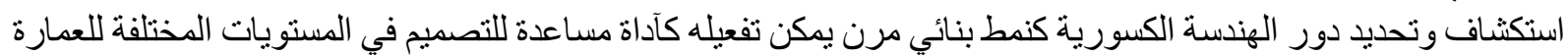
ومو اكبة لتطور لغة البرمجة في العمارة و الاستفادة منها في استحداث أنماط تتو افق مع السياق على اختلاف محتو اه ومظاهرها. 


\section{فرضية البحث}

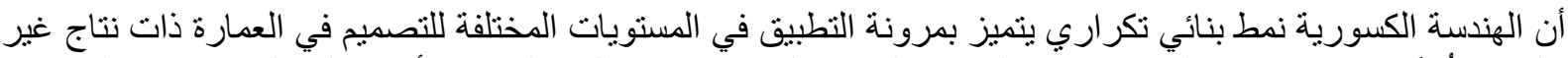

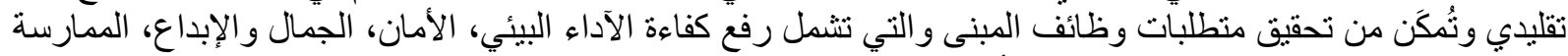

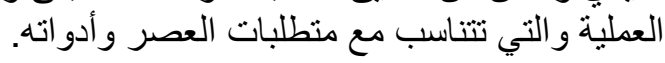

ونهجية البحثث الفحرة الفرضية يتبع البحث الخطوات التالية:

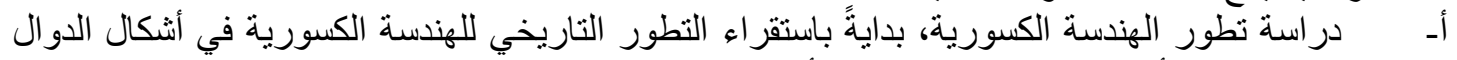

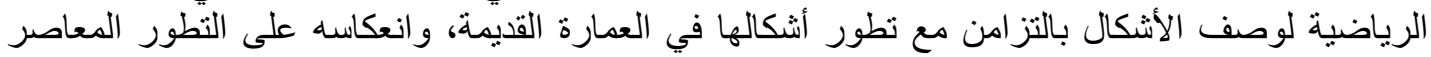

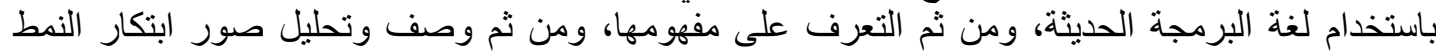

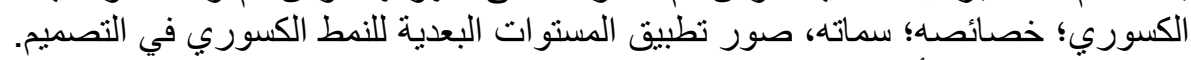

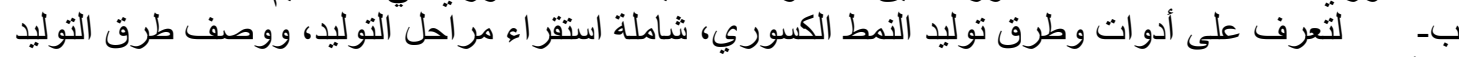

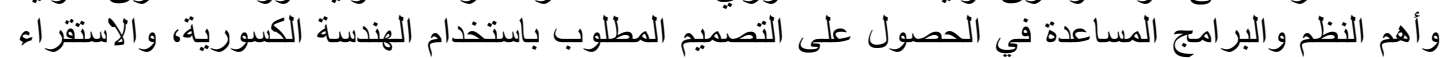

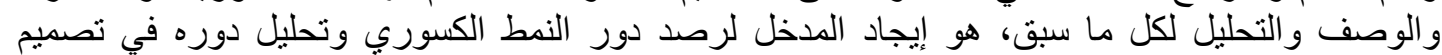
المسنويات المختلفة في العمارة.

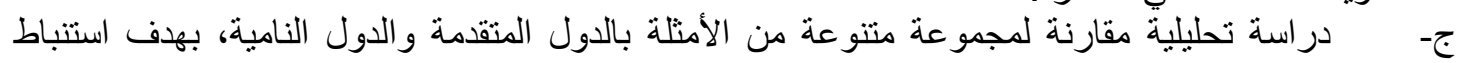
الدروس المستفادة وطرح بدائل الحلول الملائمة للسياق المعلي.

\section{ا تطور الهندسة الكسورية في العمارة}

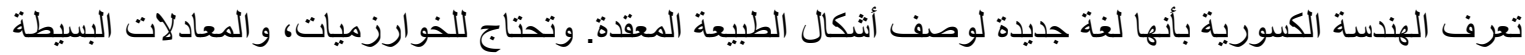

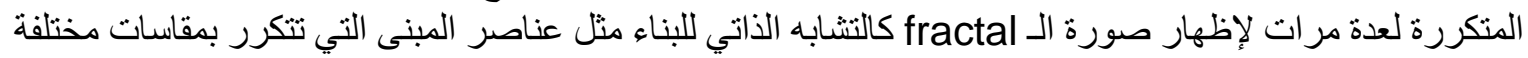

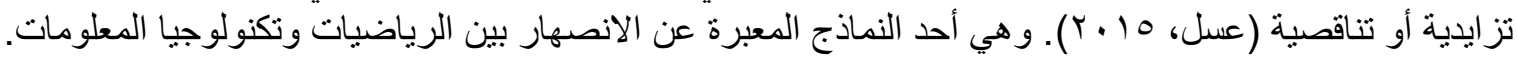

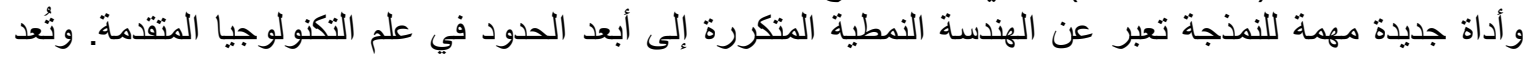

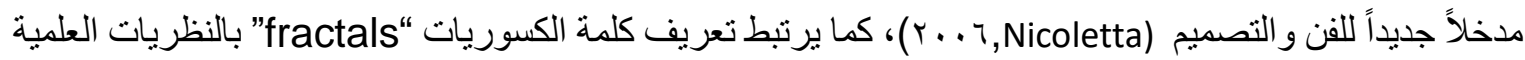

مثل التعقيد و الفوضى chaos و اللاخطية (Belma \& Ayyildiz,2016).

\section{/ إتاريخ تطور الهندسة الكسورية}

ظهرت أنماط العمارة الكسورية الأصلية في المعابد الهندوسية والكنائس القوطية، وبعض القرى الأفريقية المتماثلة ذاتباً،

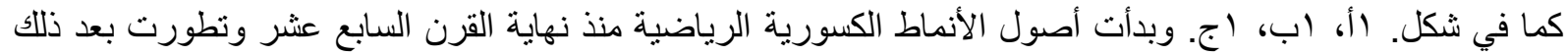

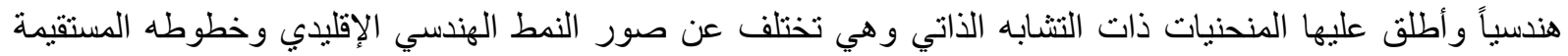

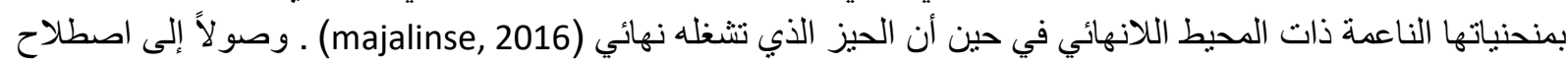

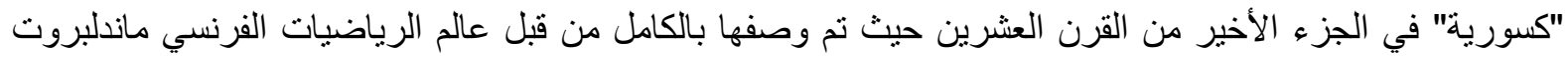

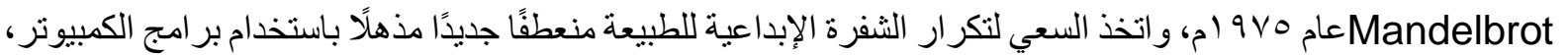

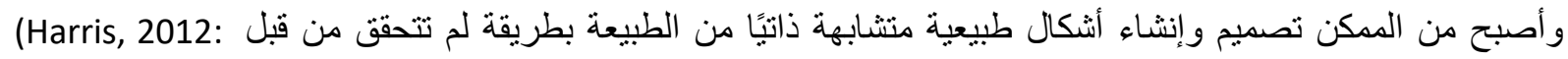

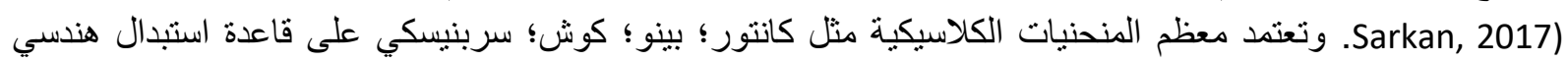

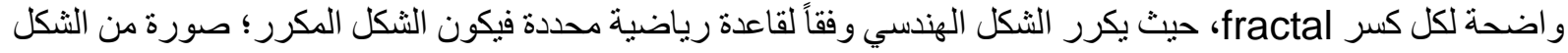

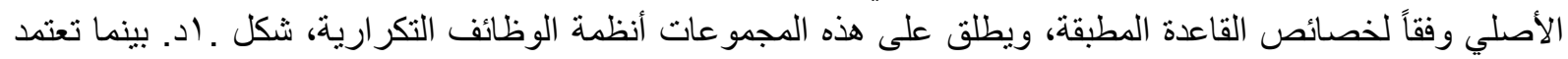

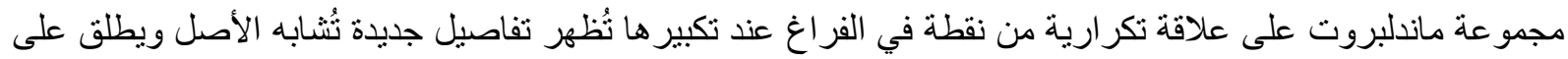

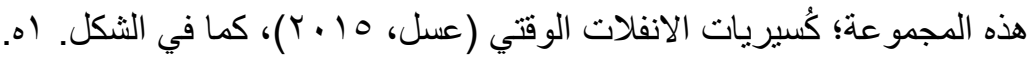


شكل (1) التطور الزمني للهندة الكسورية في أثكال الدوال الرياضية لوصف الأشكال في مقابل تطوره في العمارة قديماً

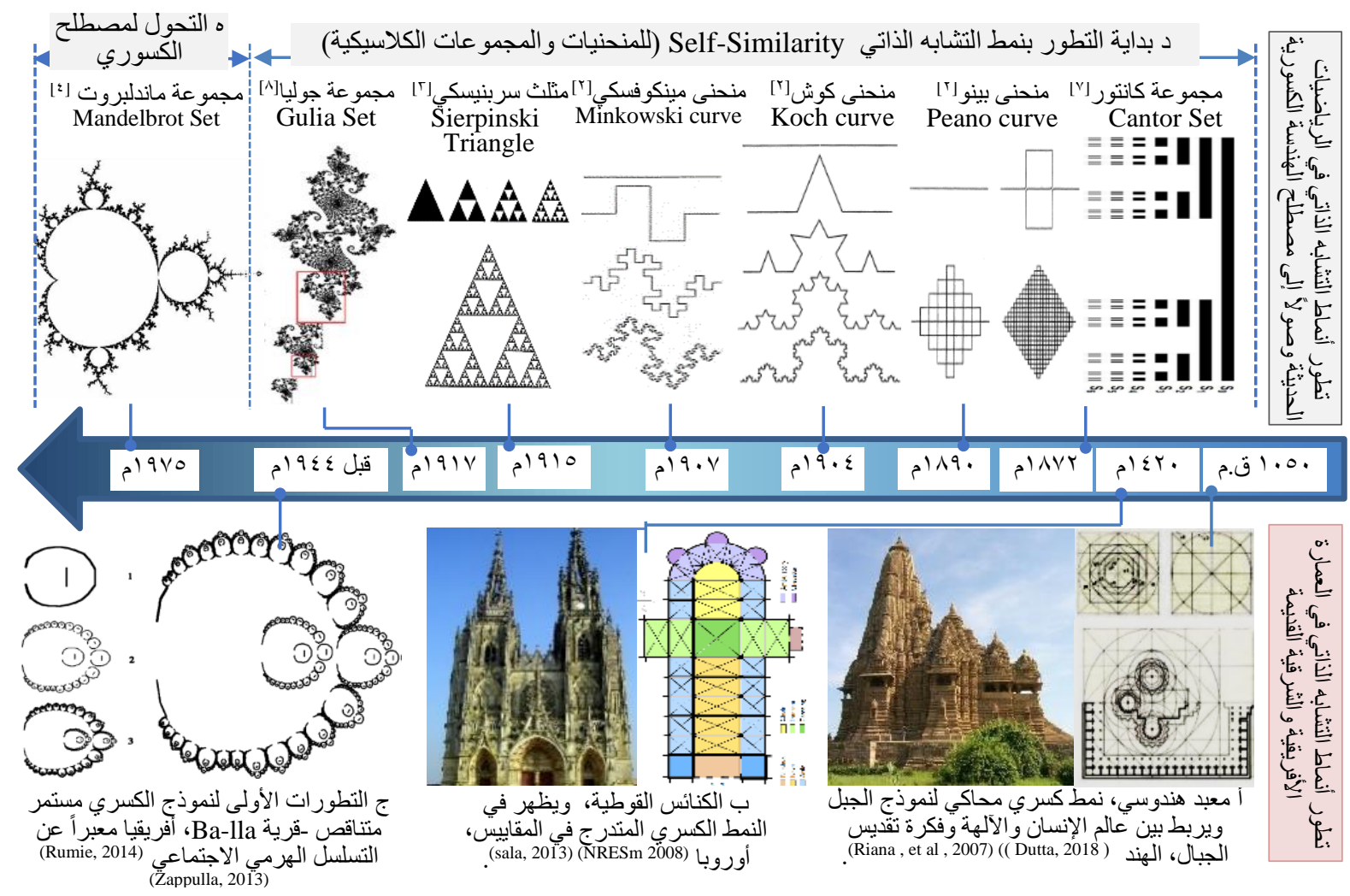

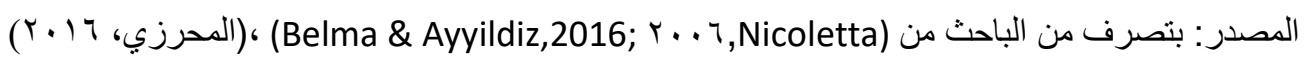

(Zappulla, 2013)

اب ابتكار الهندسة الكسورية

النكل الكسوري، شكل هندسي تقريبي أو مجزأ ويمكن تقسيمه إلى أجزاء، يكون كل منها (على الأقل تقريبًا) نسخة صغيرة

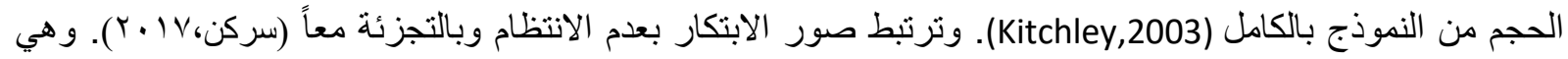

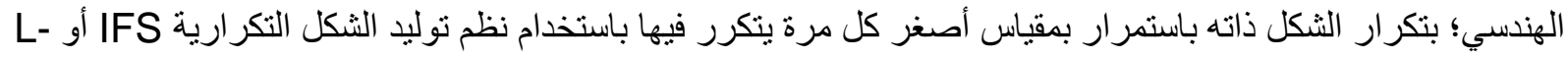

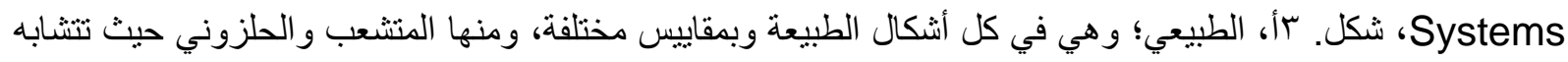

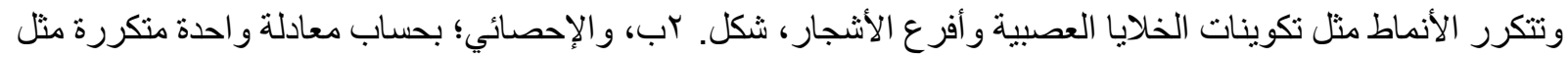
مجمو عة مندلبورت، مجمو عات جوليا، وذلك باستخدام بر امج الحاسب الآلي(

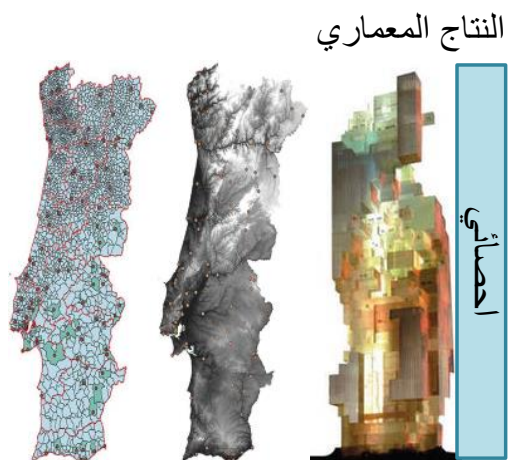

ج تمثيل كتلة المبنى احصائيا

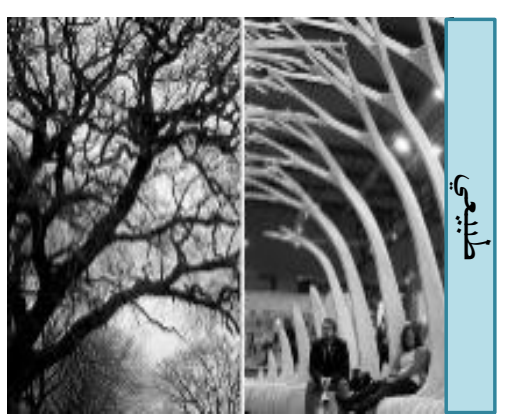

ب جناح اكسبو، ميلانو

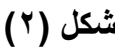

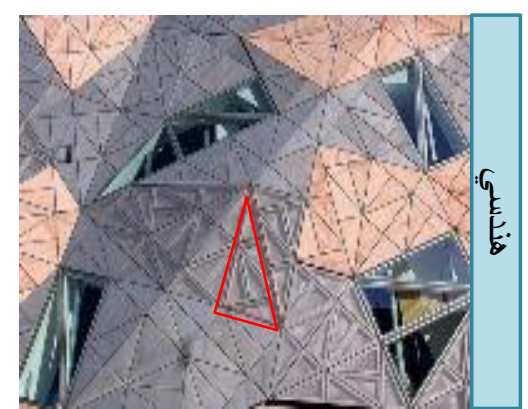

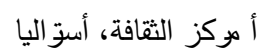

[( Fed Square.com, 2019)-(2013 , Architecture)] المصدر: بتصرف من الباحثب 


\section{r/l سمات وخصائص الهندسة الكسورية}

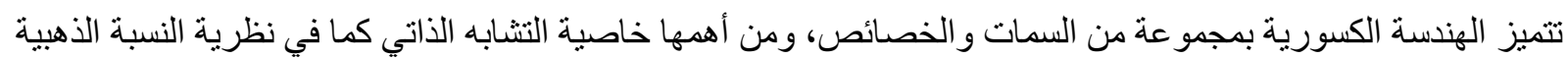

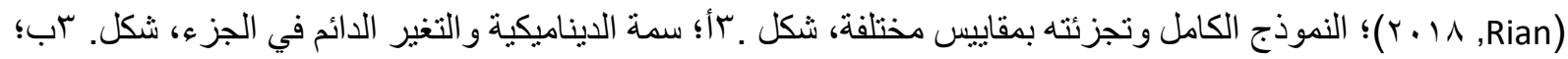

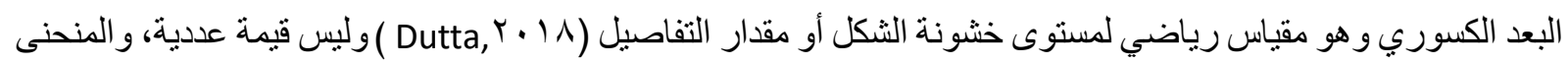

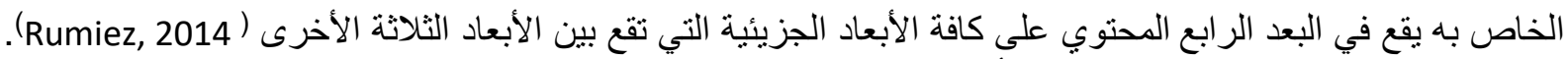

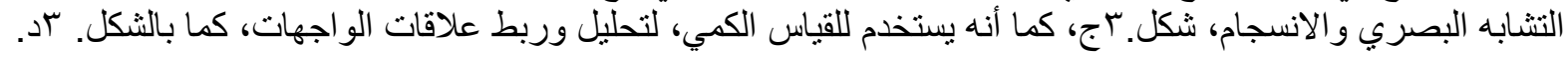

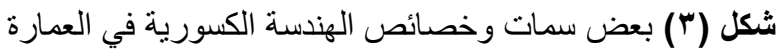
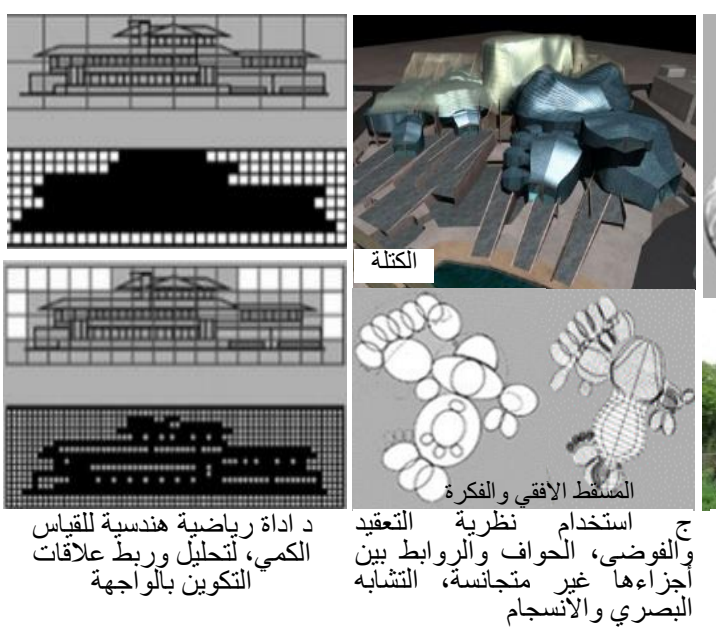

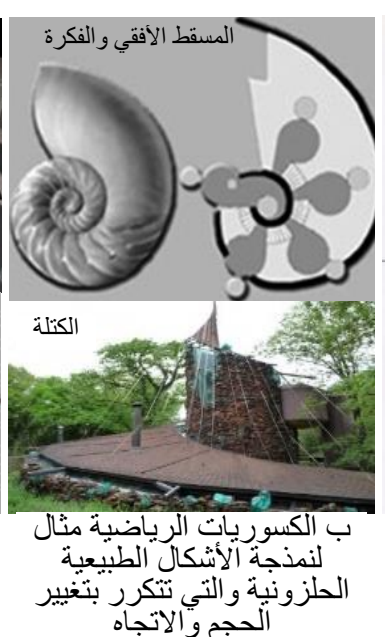

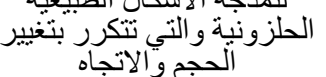

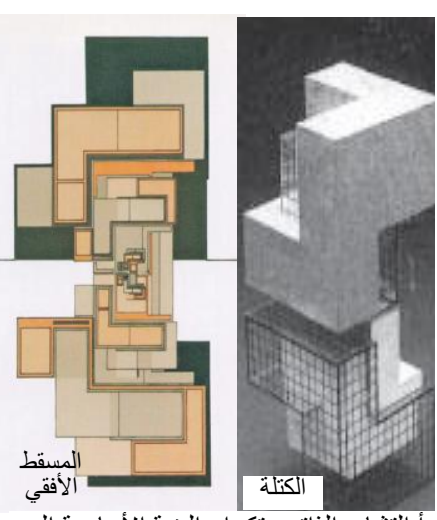

أ التثابه الذاتي: تكرار البنية الأساسية الى لى

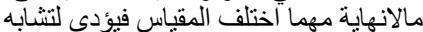
الجزء مع الكل وبزيادة التكر ار يزداد التعقيد

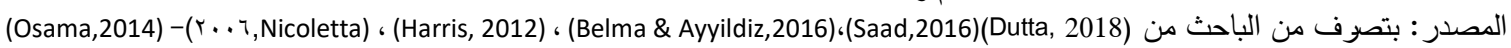

\section{/ / المستويات البعدية للنمط الكسوري في العمارة}

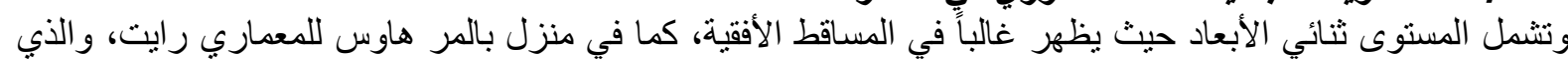

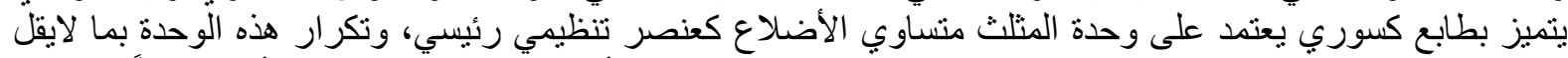

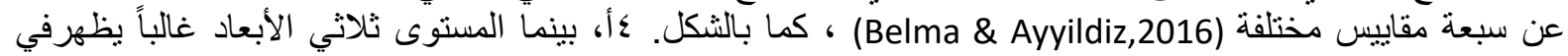

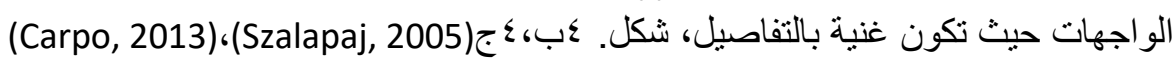
شكل (؛ ) المستويات البعدية للنمط الكسوري في العمارة

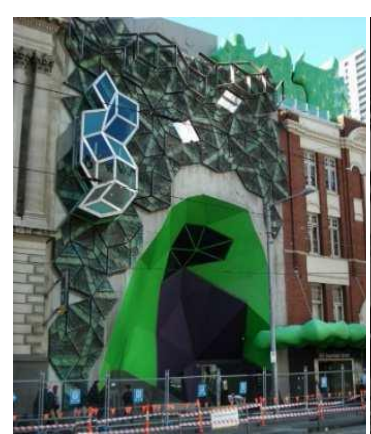

ب نموذج واجهة كسورية ، ج معهد للتكنولوجيا، أستر اليا

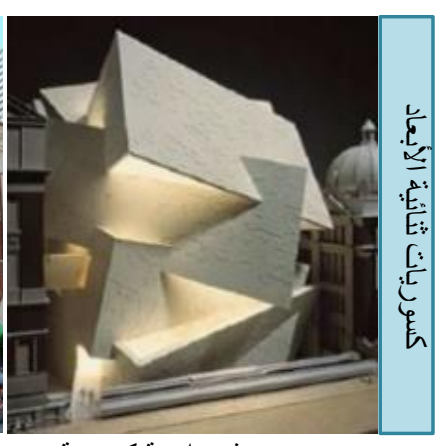

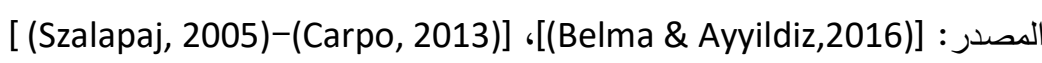

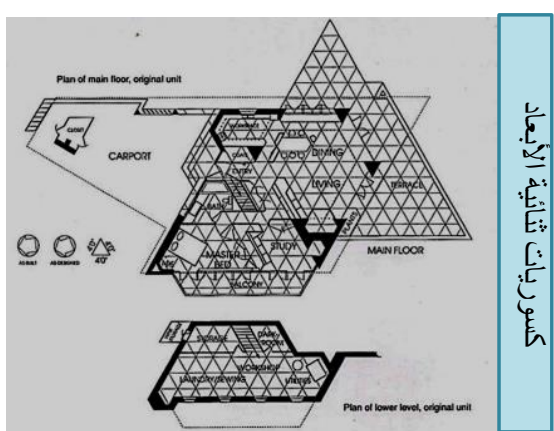

ابالمر هاوس، مينتيجان

r طرق وأدوات توليد النمط الكسوري بمساعدة تطبيقات وبرامج الحاسب الآلي

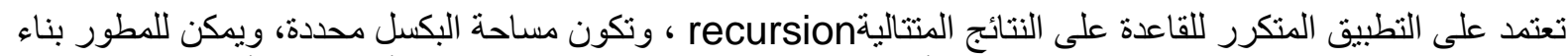

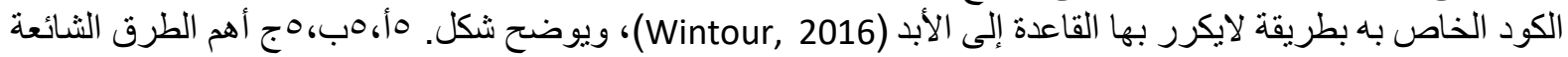
لتوليد النمط الكسوري. 
شكل (•) أهم الطرق الثنائعة لتوليد النمط الكسوري في العمارة
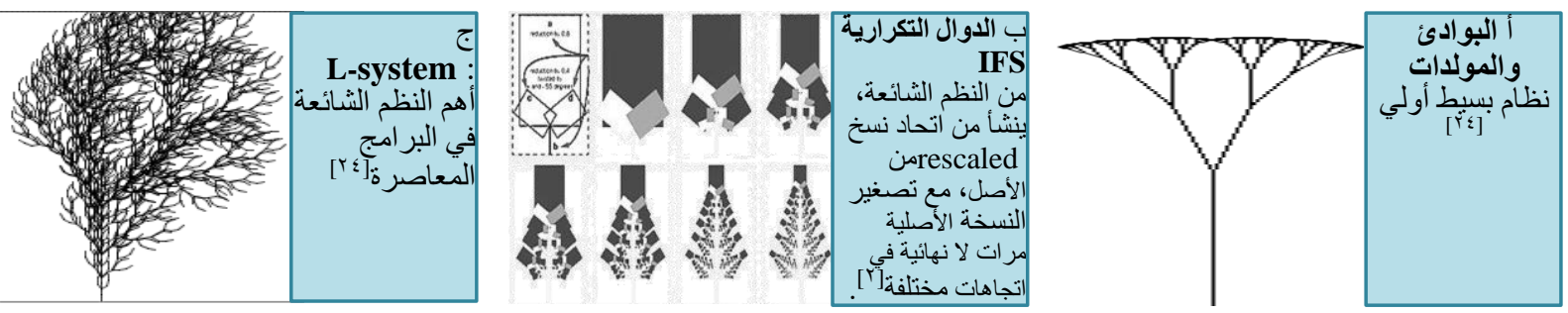

المصدر: [Wintour, 2016] [2006,Nicoletta]

\section{1/r مراحل عملية توليا النمط الكسوري}

( ) خلق شكل معين: البادئ initiator و هو شكل البداية؛ (r) اختيار قاعدة التوليد rule لكيفية تغيير هذا الثكل: استبدال

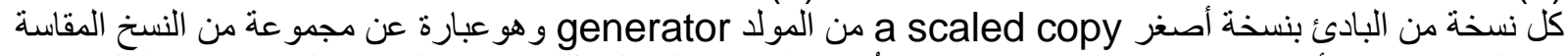

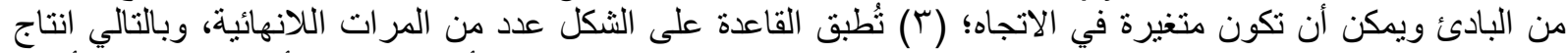
الثكل باستخدام الهندسة الكسورية (Efe, 2016). وتستخدم هذه العملية لإنشاء مسقط أفقي، واجهة، أو شكل ثلاثي الأبعاد

.(Flake, 2000)

\section{r/ץ البرامج والنظم الرقمية المعاصرة لتوليد النمط الكسوري}

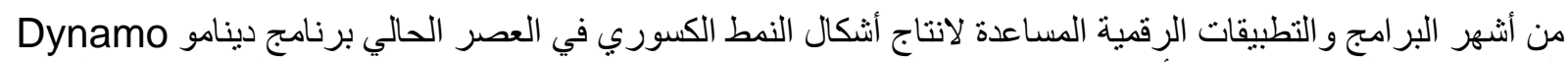

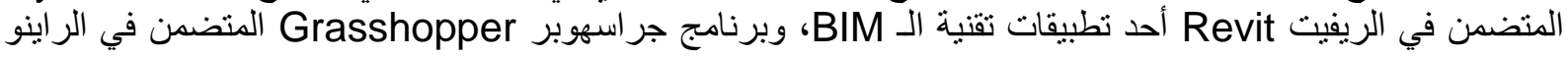

Rhinoceros

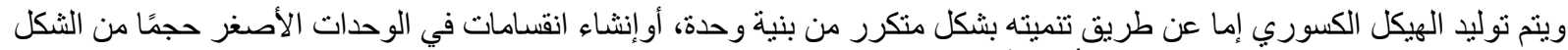

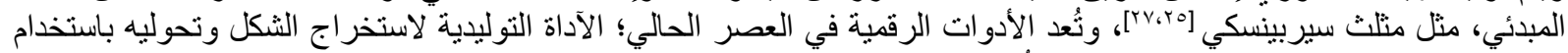

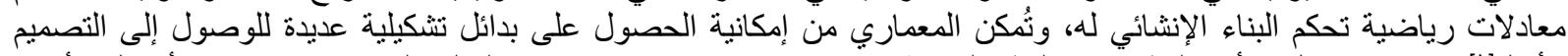

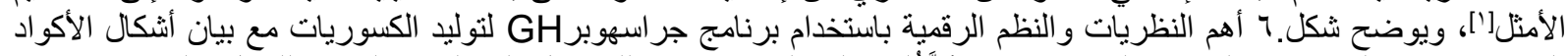

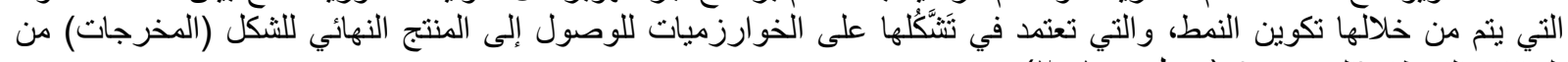

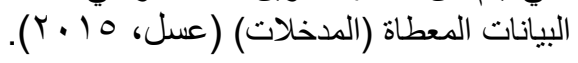

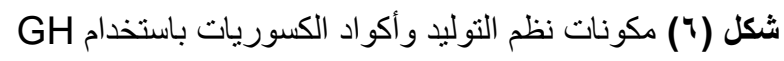

GH Sكونات النظم الرقمية لتوليد النمط الكسوري داخل

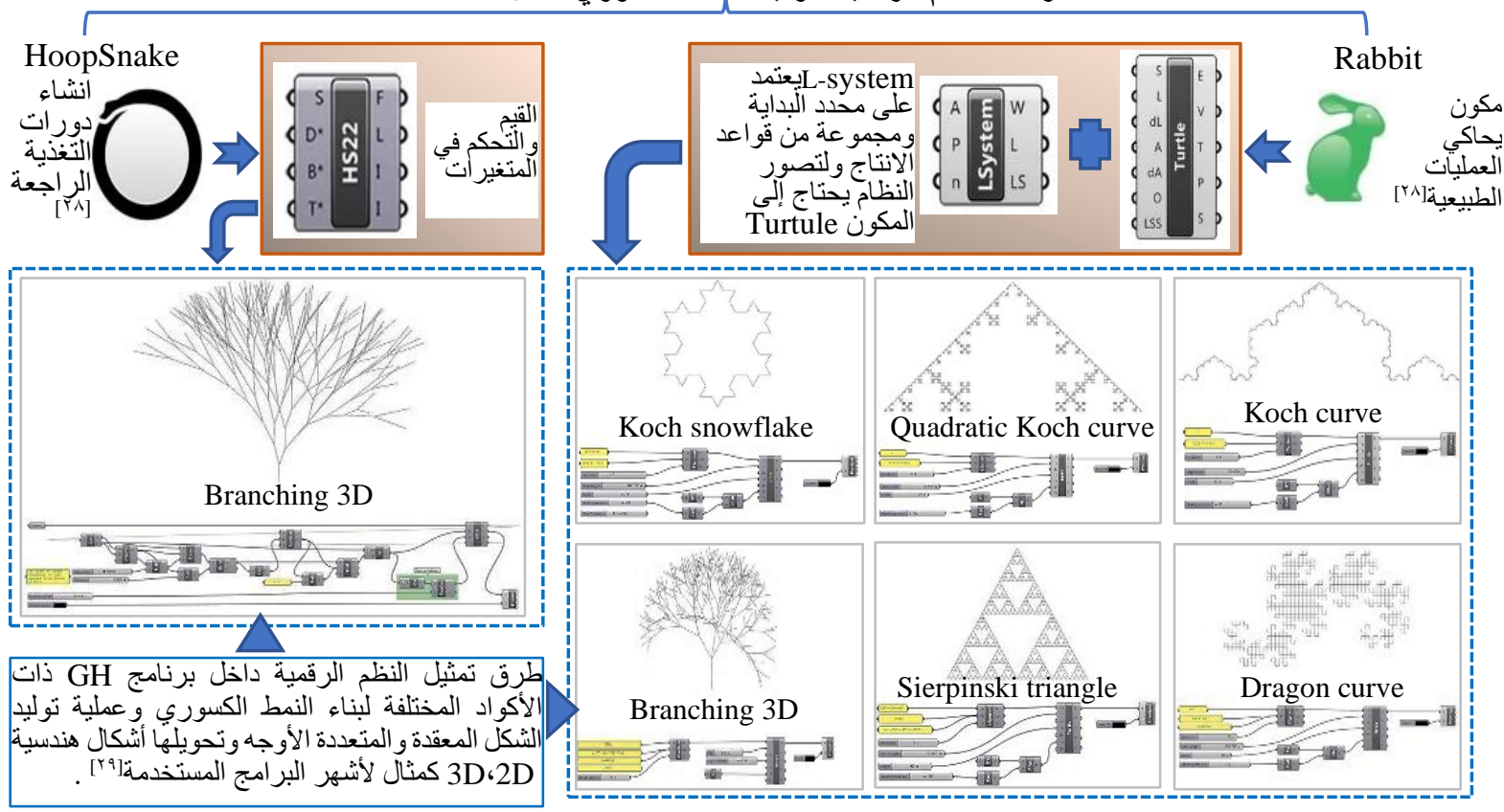

[( Wintour, 2016)،(Shiffman, 2012) [المصدر : بتصرف من الباحث 


\section{r تحليل مستويات تطبيق نمط الهندسة الكسورية كوحدة بنائية للتصميم في العمارة}

ارتبط تطوير نظريات الكسوريات والفوضى و التعقيد والخوارزميات، بتطوير البرمجيات لنترجم هذه النظريات إلى أنثكال

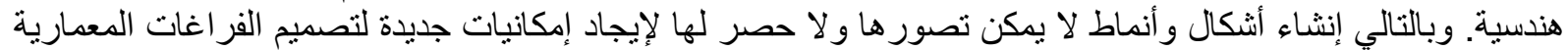

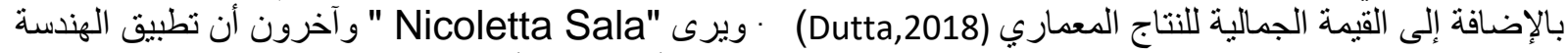

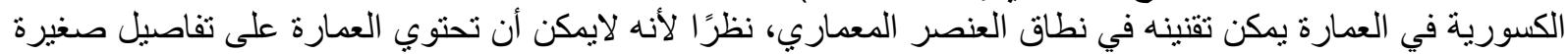

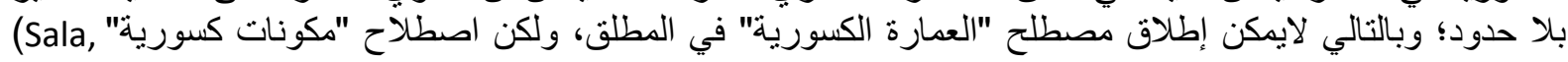

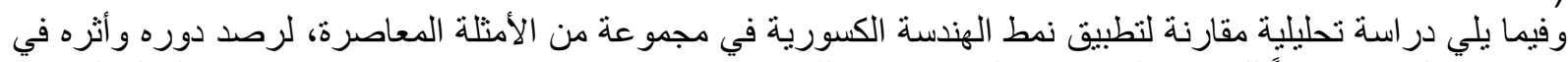

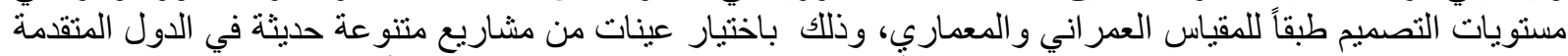

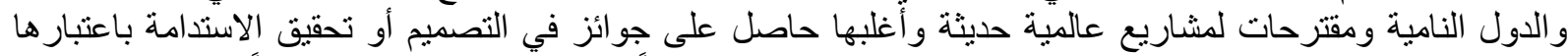

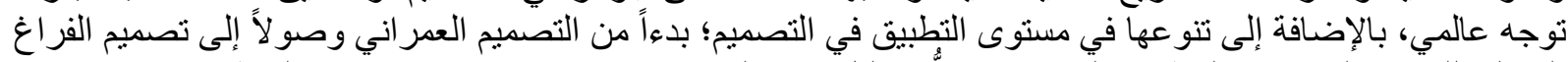

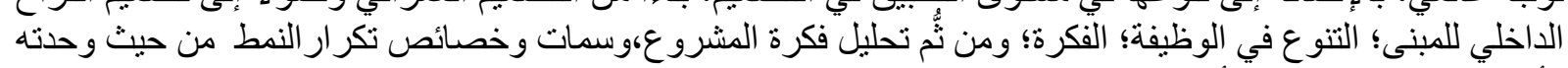

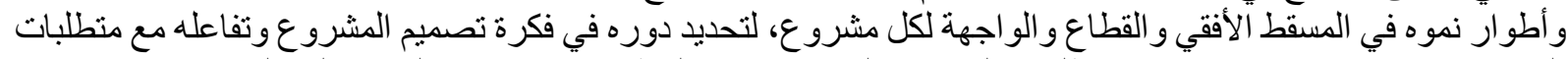
العصر ، ومدى تحقيقه لاعتبارات ومتطلبات العات العمارة و العمر ان سواء الوظيفية؛ الانشائية؛ البيئية؛ الجمالية.

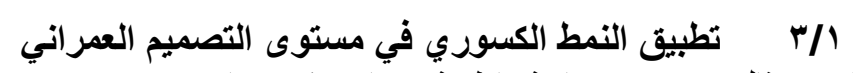

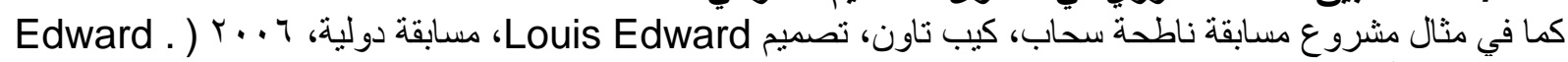

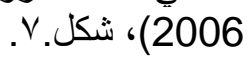

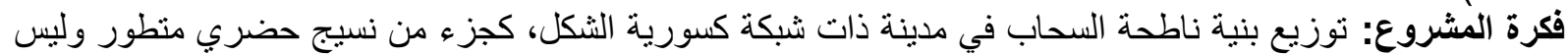

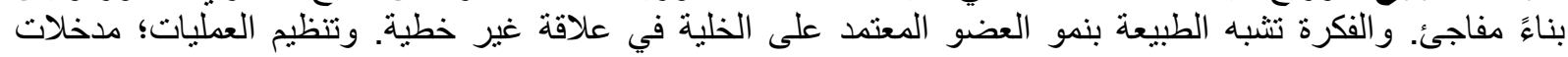

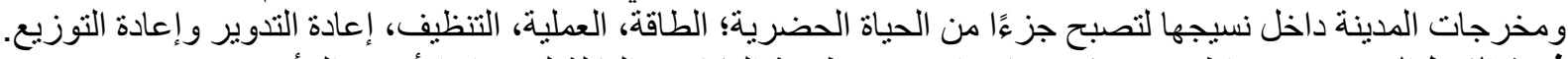

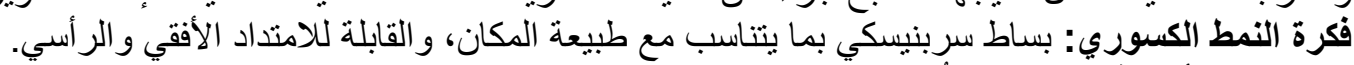

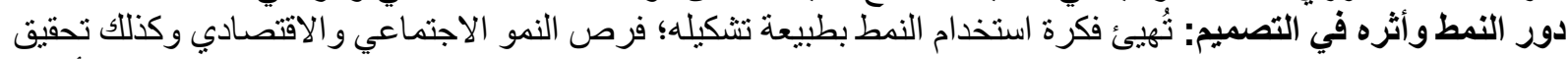

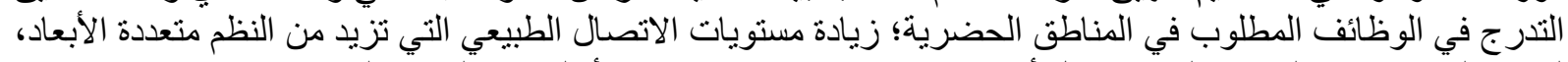

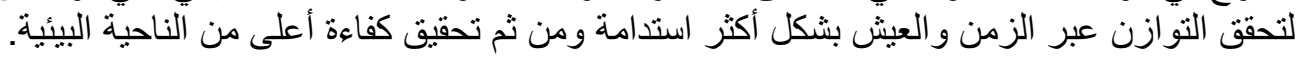
شكل (V) يوضح الهندسة الكسورية كنمط بنائي للتشكيل الحضري، مدينة كايب تاون.

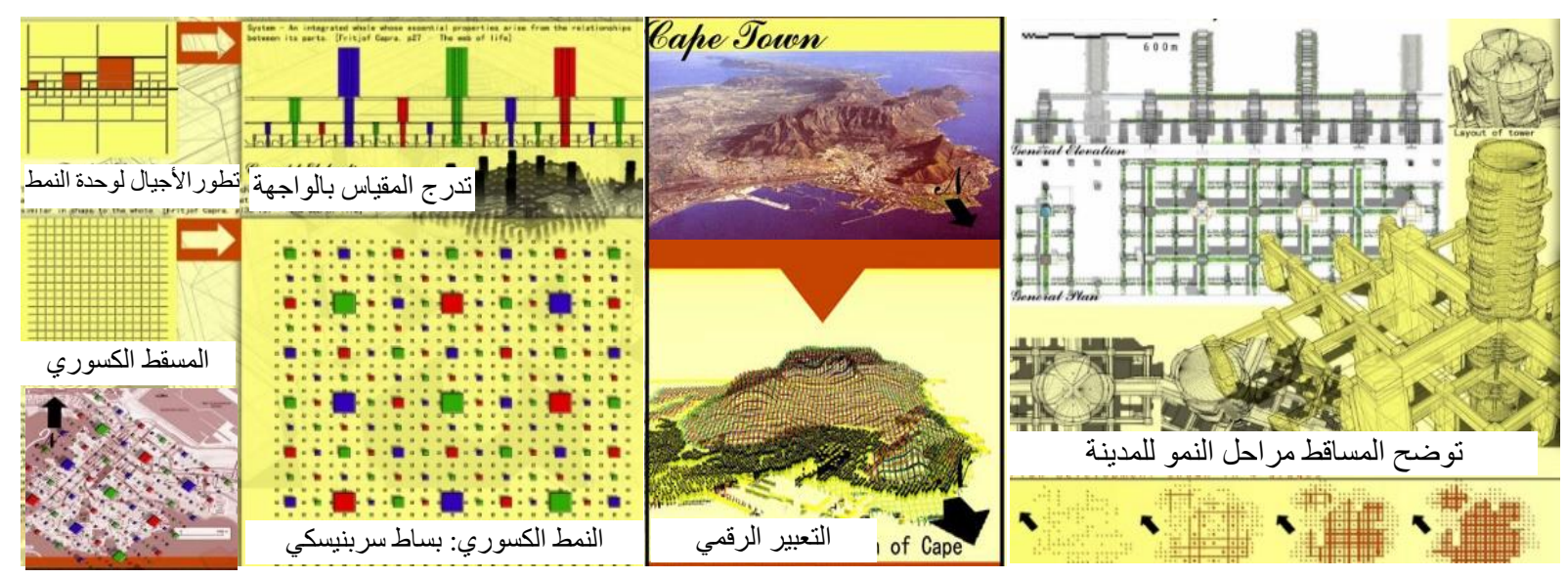

[Edward . 2006] [المصدر:

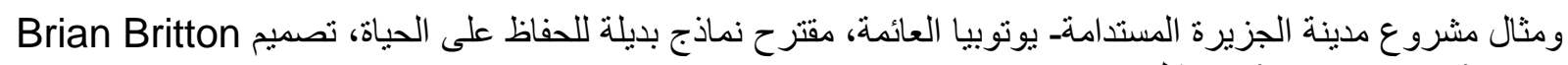

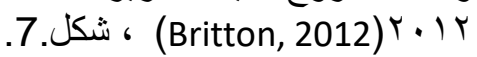

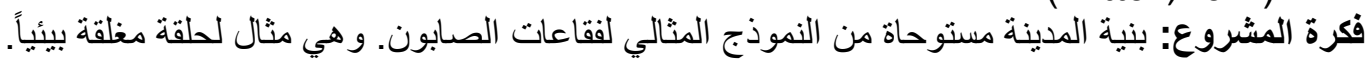

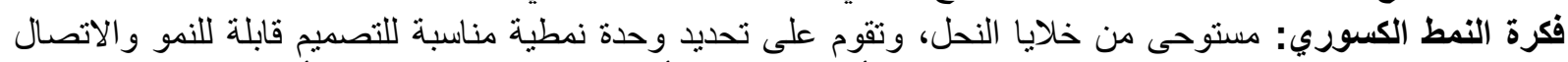

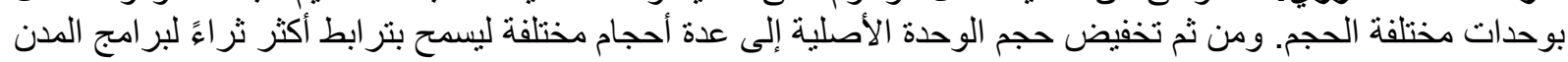

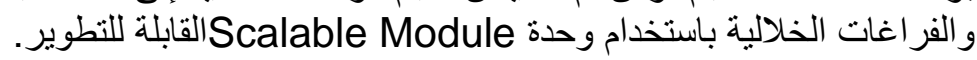


؛ دور النمط وأثره في التصميم: كل وحدة نمطية تحوي برنامج متعدد الاستخدام بما يتناسب مع الوظائف المختلفة. ويتيح

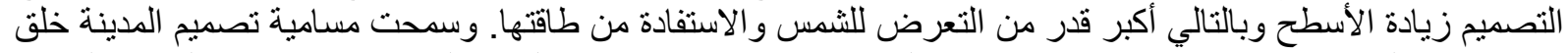

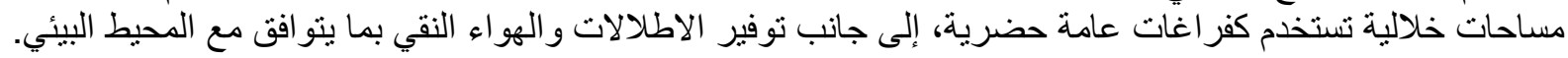
شكل (^) يوضح الهندسة الكسورية كنمط بنائي للتنكيل الحضري، الدينه الدينة العائمة.

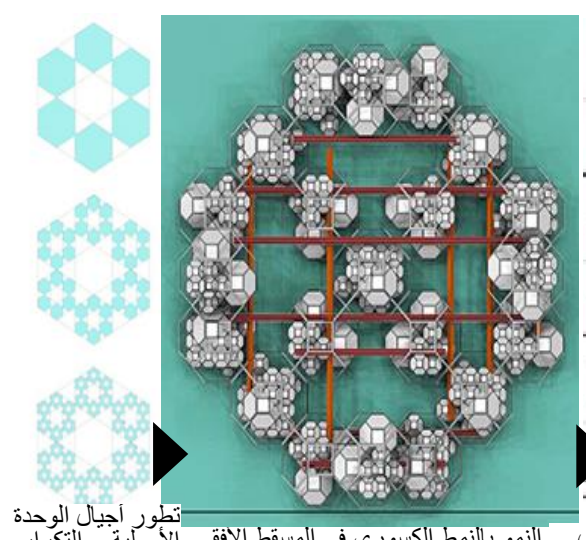

النمو بالنمط الكسوري في المسقط الافقي الاصطور اجيال التوحدة سربنيسكي مثلث

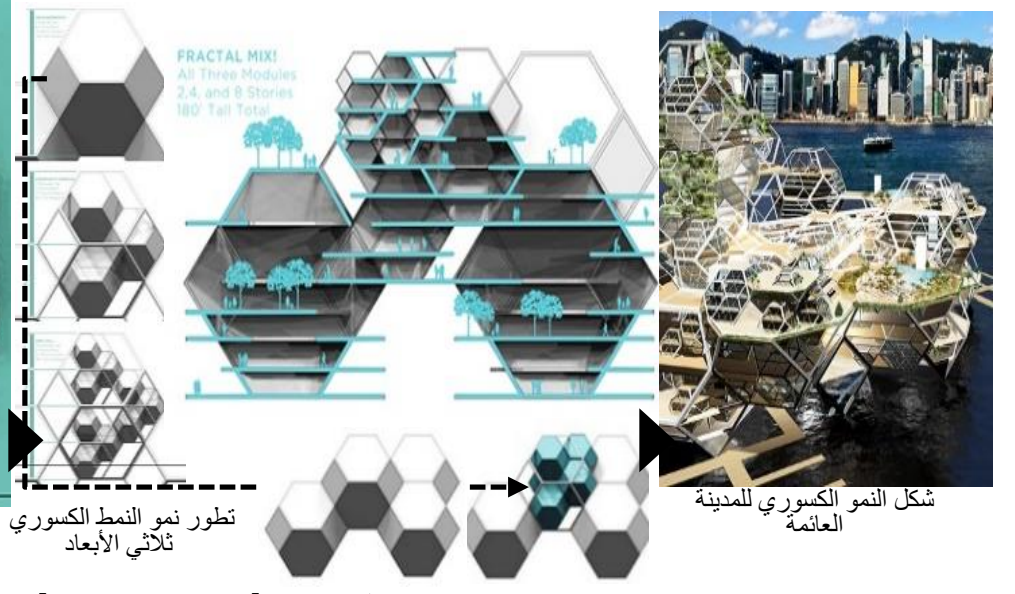

[المصدر: [Britton, 2012].

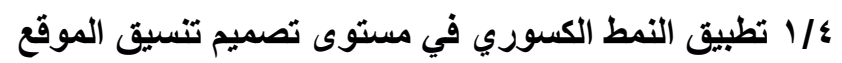

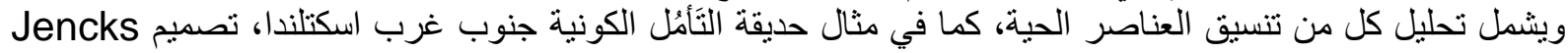
Charles فكرة المشروع: تصميم المناظر الطبيعية التي من شأنها أن تربط بين عالم الفن و الطبيعة والعلوم، وهي عبارة عن تلال من

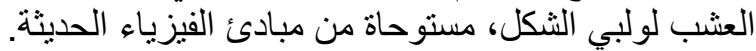

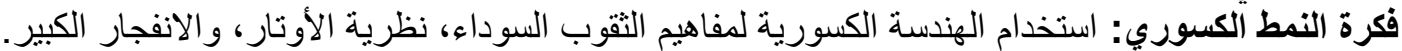

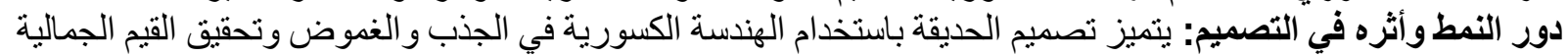
و التشكيلية المطلوبة في هذا النوع من المشاريع.

شكل (9) يوضح الهنسة الكسورية كنمط بنائي لتصميم تتسيق الموقع، حديقة الضاربة الكونية
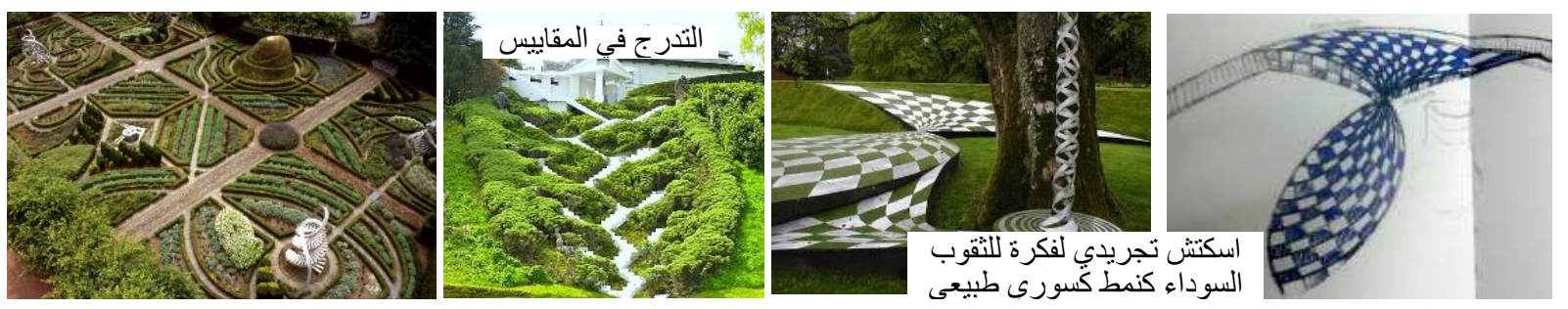

المصدر: [Jencks, 2011].

وتحليل تنسيق العناصر الغير حية، كما في جناح MadeExpo بغابة موناليزا الكسورية، تجربة تم تنفيذها سنة 20112 (Jencks, 2011)

فكرة المشروع: استلامام من شجرة الحور لتتحول تدريجياً من نبات صغير إلى شجرة خشبية معمرة وتتكرر لتصنع الغابة.

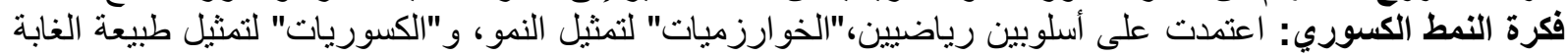

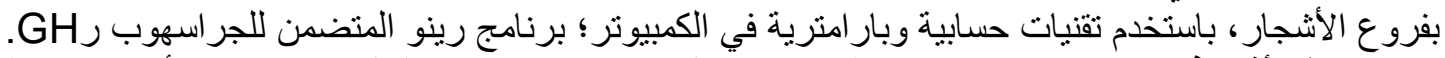

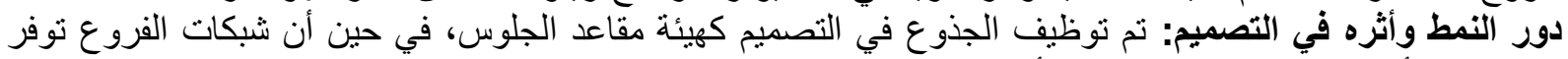
الثعور بالمأوى وتحقيق الظلال. بينما الأشكال المنحنية تعبر عن معنى الطبيعة. 
شكل ( • (1) يوضح الهندسة الكسورية كنمط بنائي لتصميم تتسيق الموقع، جناح: MadeExpo

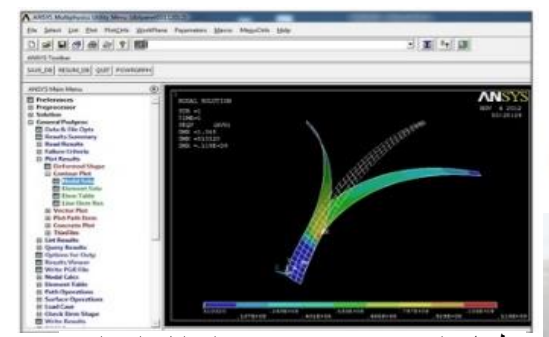

لبناء الجناح استخدام برنامج التحليل الهيكلي ANSYS
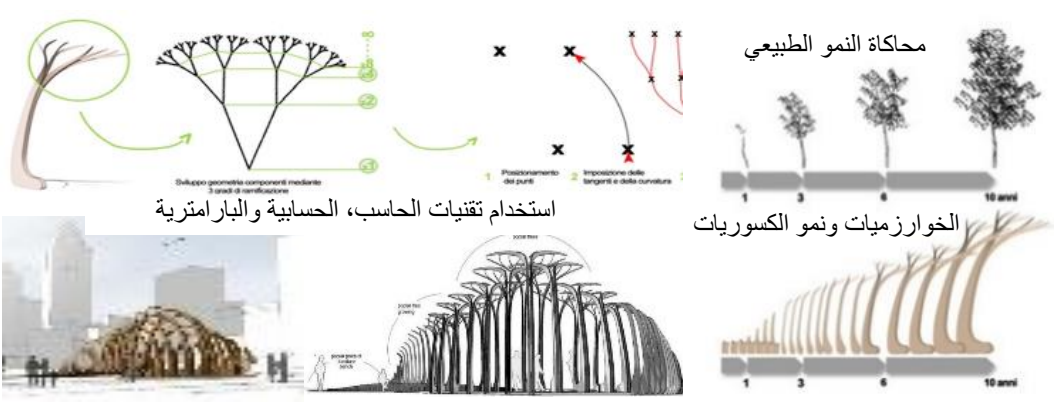

[Architecture, 2013] : المصدر

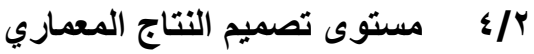

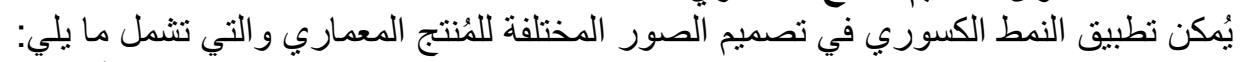

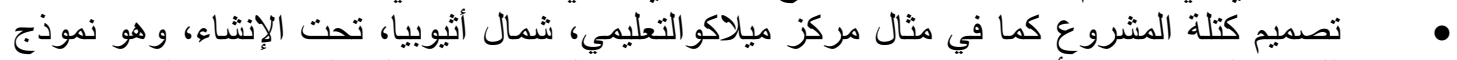

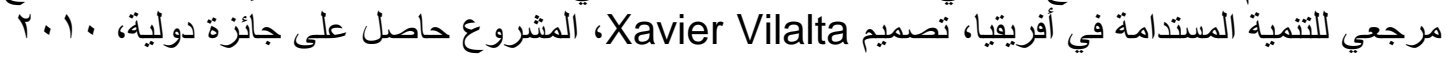

. (ArchDaily, 2009)

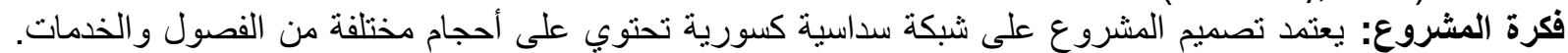

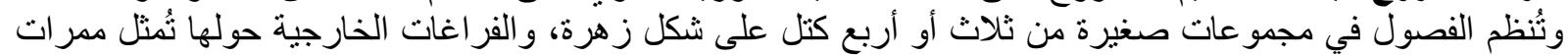
المدرسة. فكرة النمط الكسوري: مستوحى الثكل الطبيعي للزهرة، ومن العمارة الأفريقية التقليدية الثائعة باستخدام النمط الهندسي

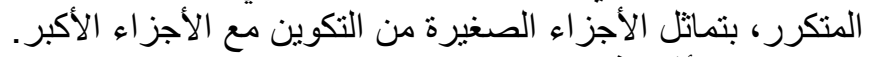

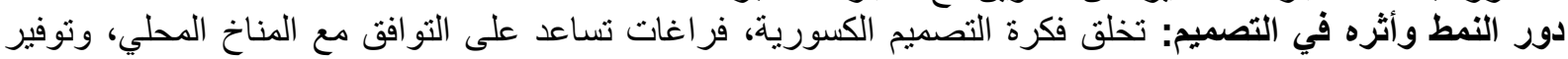
التهوية الطبيعية لفر اغات التعلم من كلا الجانبين. وبالتالي تحسين الآداء البيئي باستخدام فكرة مبتكرة تحقق ديناميكية للفر الغ الغئ.

$$
\text { شكل (1' (1) يوضح الهندسة الكسورية كنمط بنائي لتصميم الكتلة، مركز تعليمي بأثيوبيا }
$$
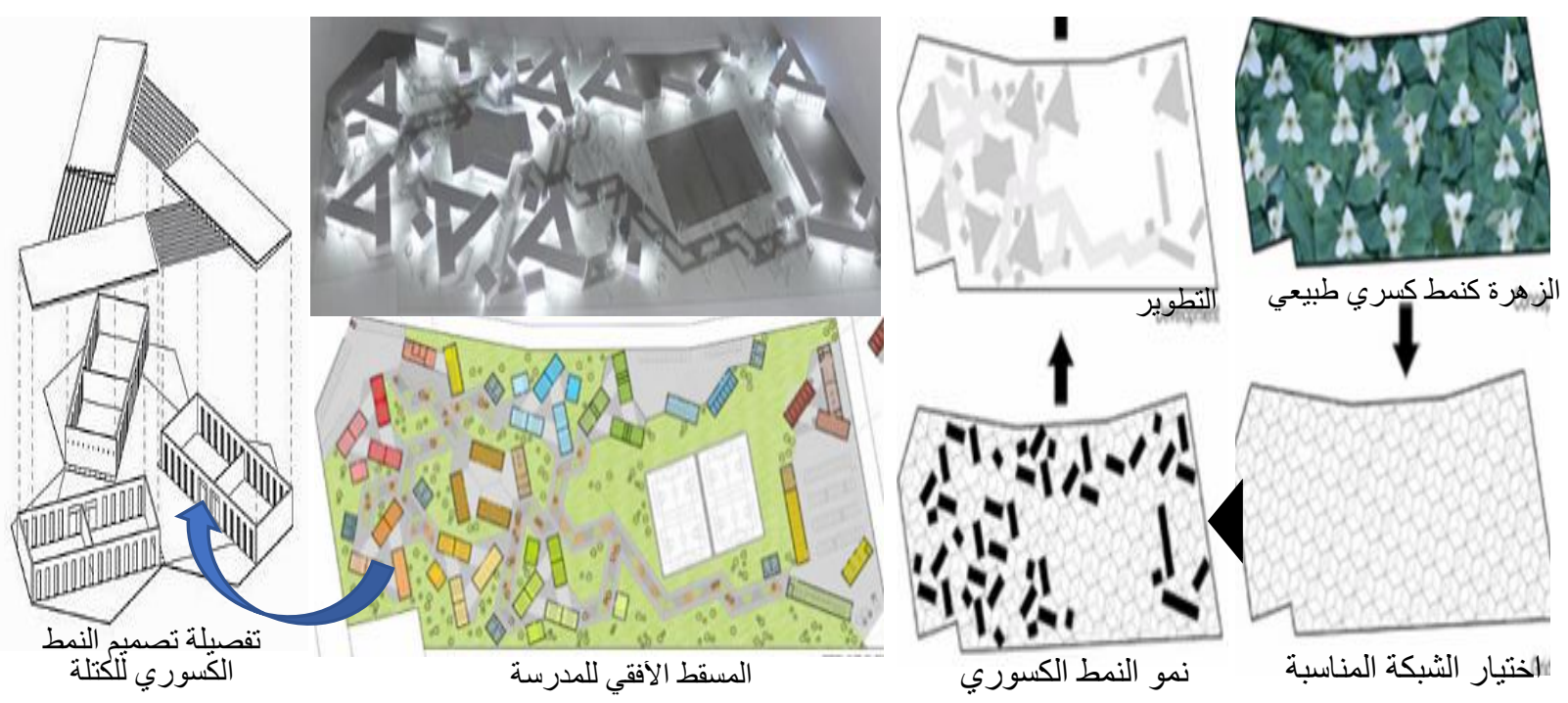

(ArchDaily, 2009) : المصدر)

تصميم عناصر الكتلة ويشمل تحليل أفكار العناصر المكونة للكتلة، مساقط أفقية ور أسية، الو اجهات، باستخدام النمط

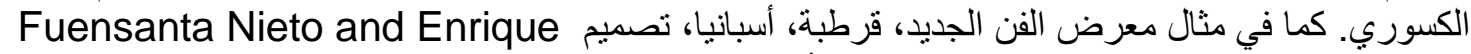

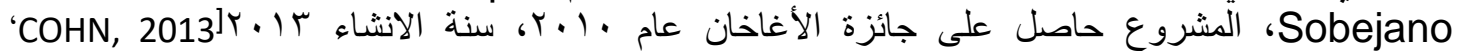

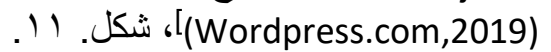

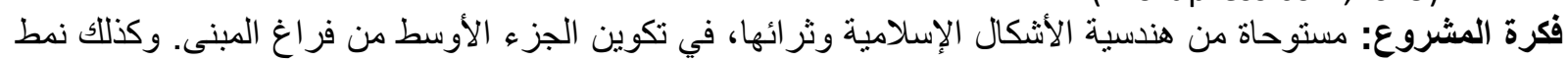

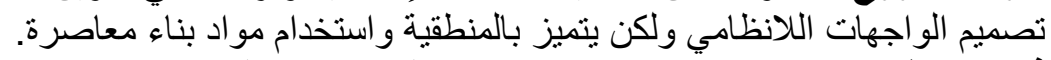

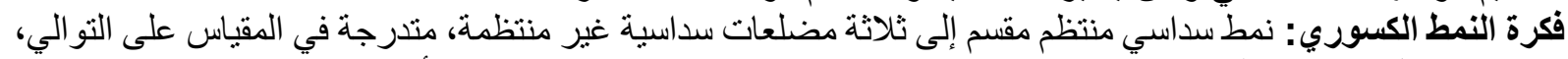

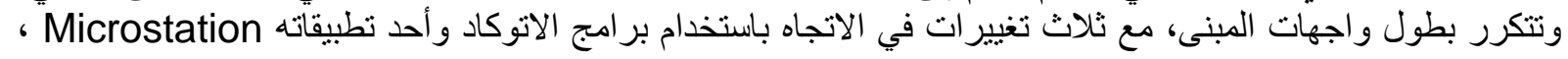




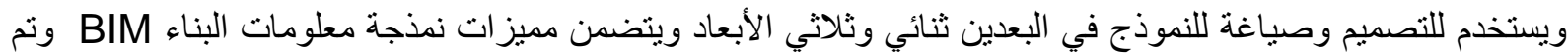

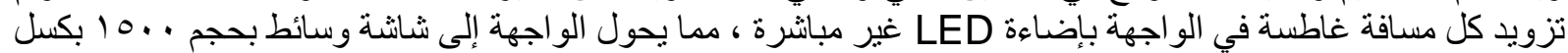

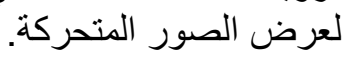

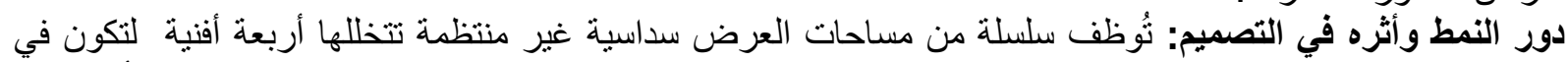

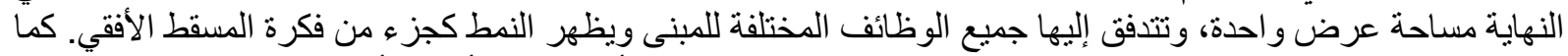

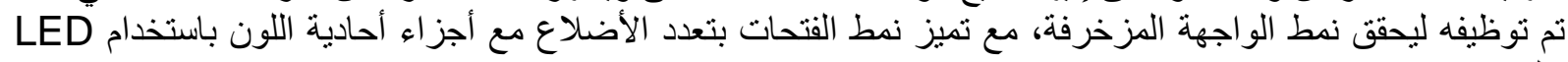
خلفها.

ومثال مركز التسوق بأديس أبابا، أثنيوبيا، تصميم Vilalta Architects ، و والحاصل على جائزة اليونسكو الخاصة

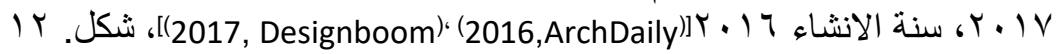

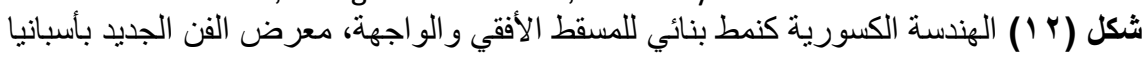
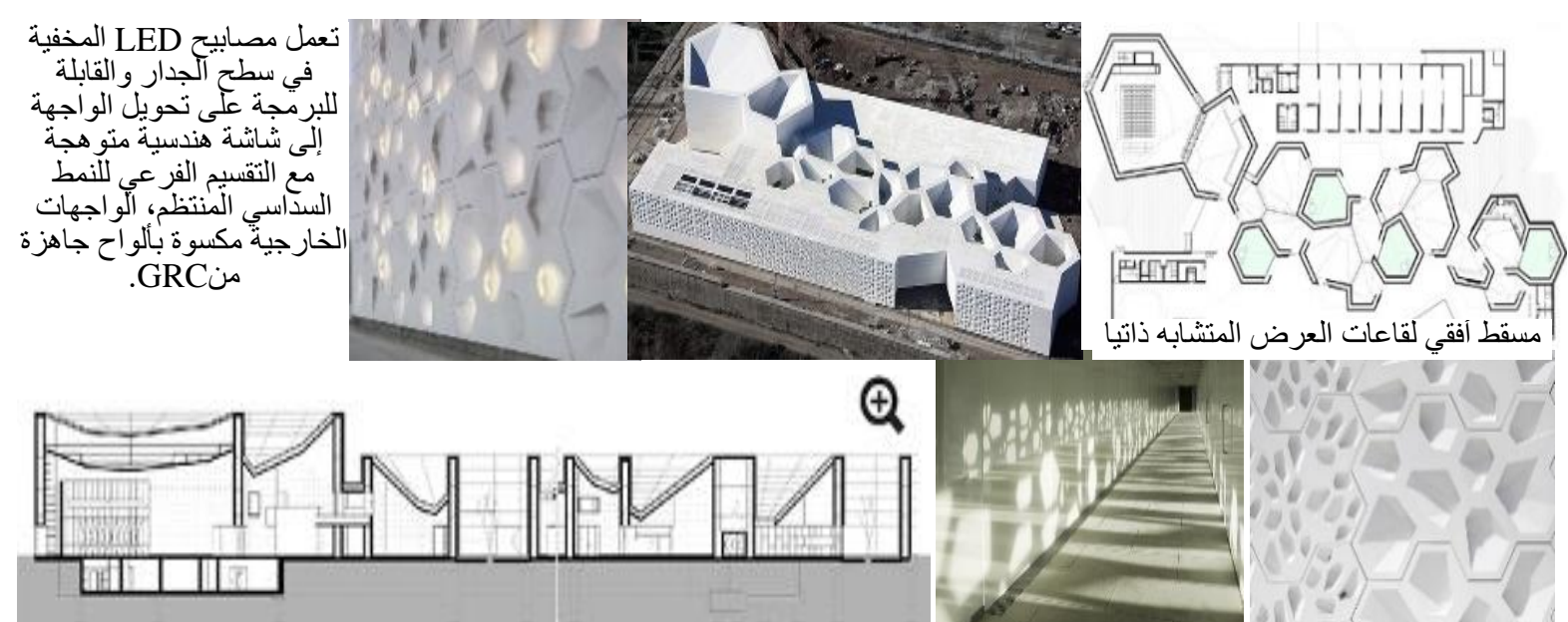

المصدر : [ COHN, 2013،(Wordpress.com,2019)

فكرة المشروع: الحفاظ على التقاليد المحلية بشكل جديد يمكن نقلها للأجيال القادمة ومن ثم المثل نقله إلى المستقبل.

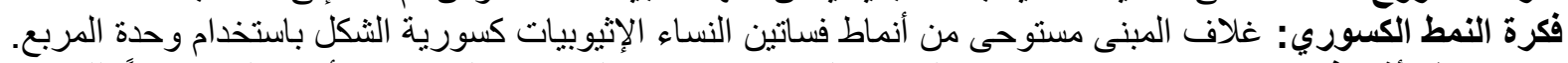

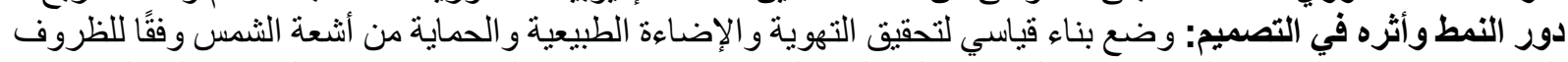

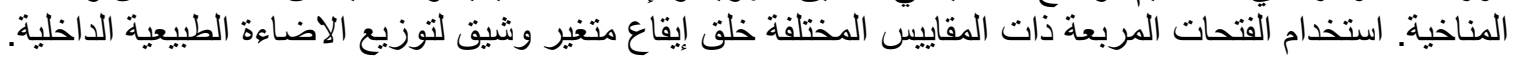

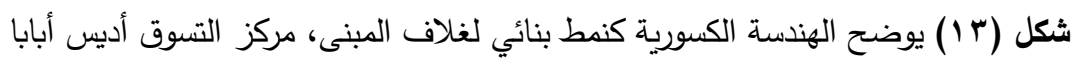

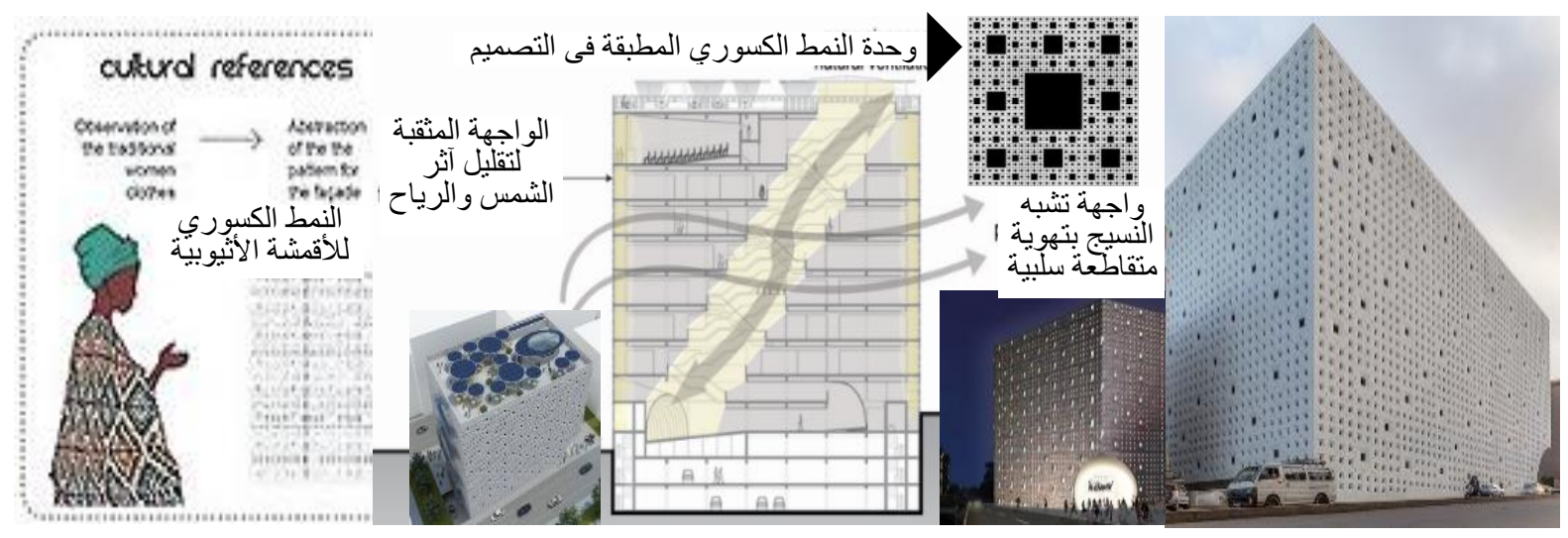

[ (Archdaily, 2018)،(Designboom, 2017)] المصدر

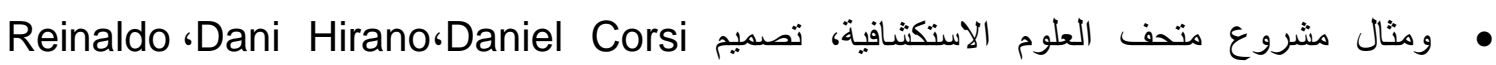

Nishimura

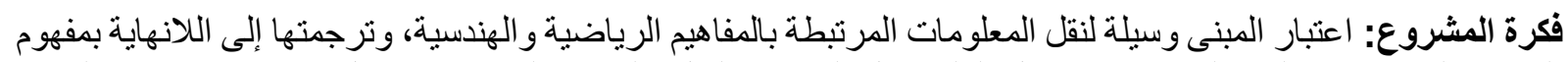

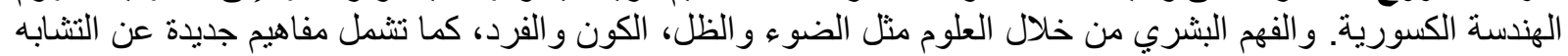

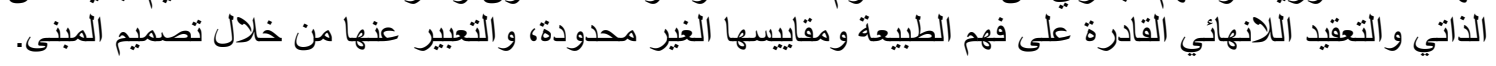




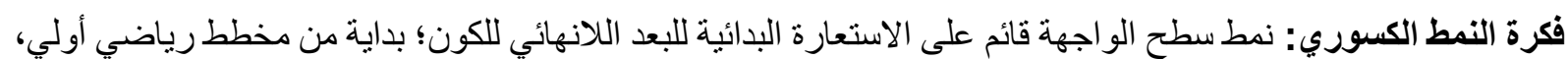

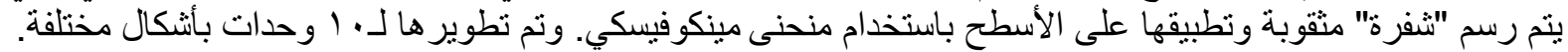

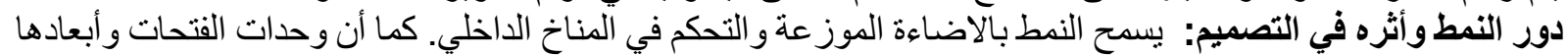

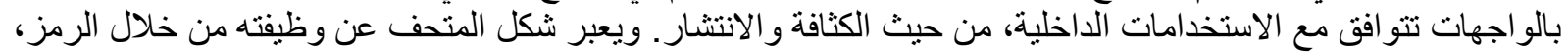
و الحماية المناخية، الظو اهر الكونية، فكرة اللانهاية، تحفيز خيال الفرد ومعرفته عن العلاقة بين الإنسان و الطبيعة و العلوم.

شكل (؟ ) يوضح الهنسة الكسورية كنمط بنائي لتصميم الواجهات، منحف العلوم الاستكثافية، البرازيل.

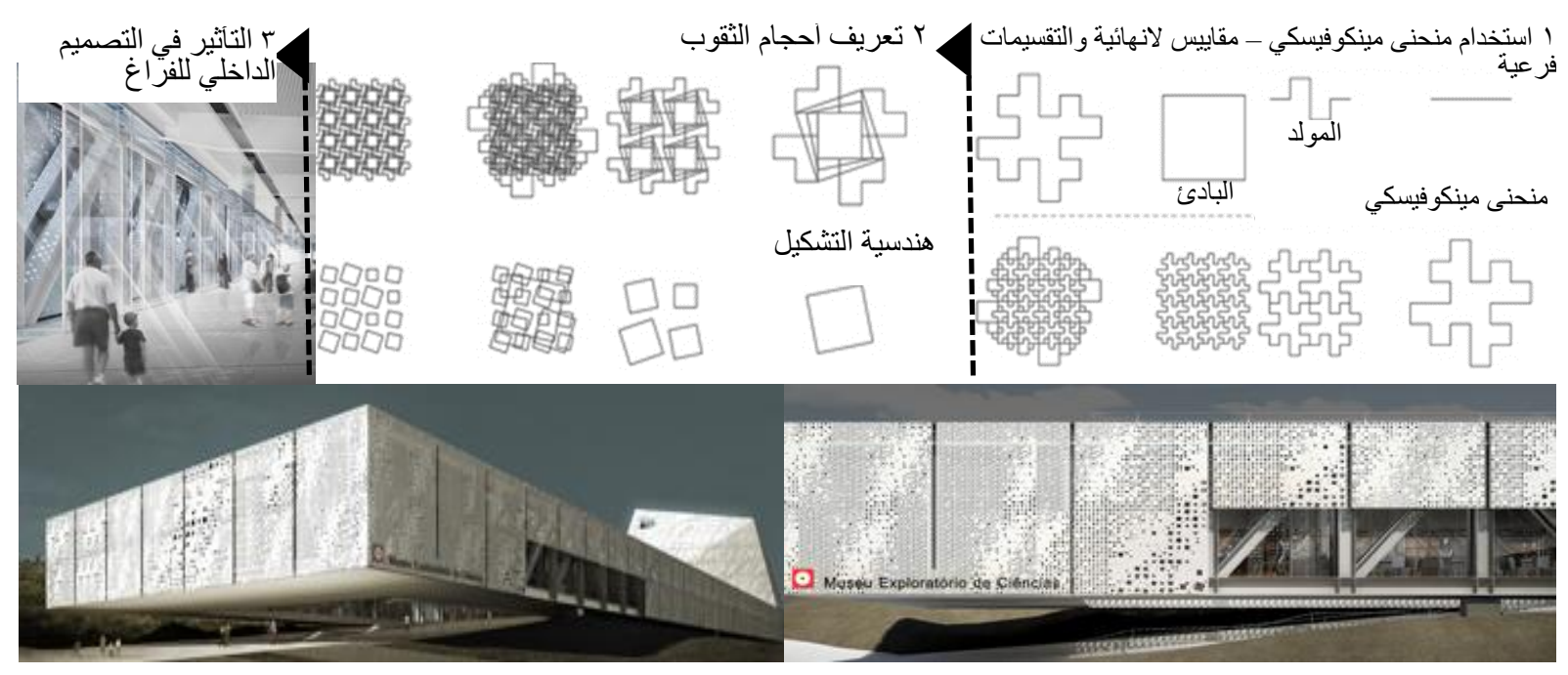

[2009 Byng, : المصدر

• تصميم الفراغ الداخلي ومكوناته مثل مشروع صالة r) مطار شاتراباتي شيفاجي الدولي في مومباب، تصميم Skidmore, owings \& merrill (SOM)

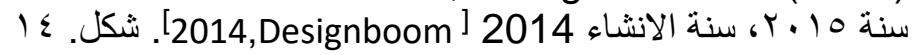

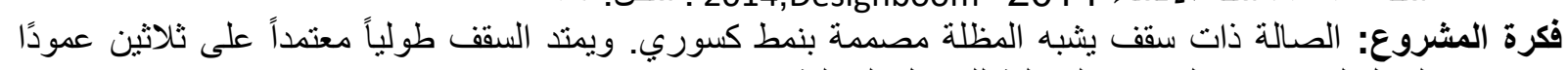

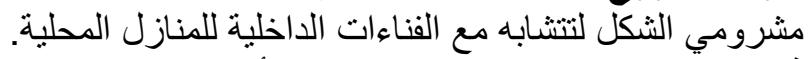

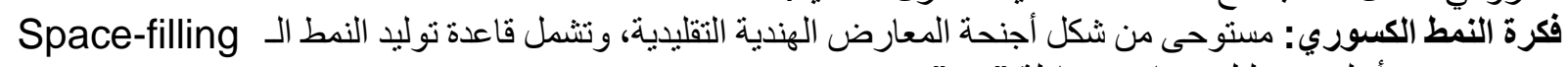

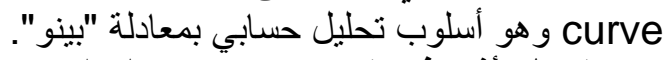

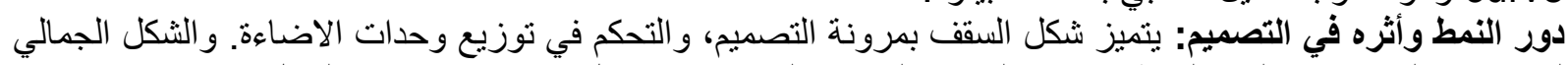

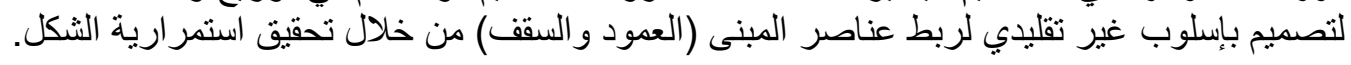

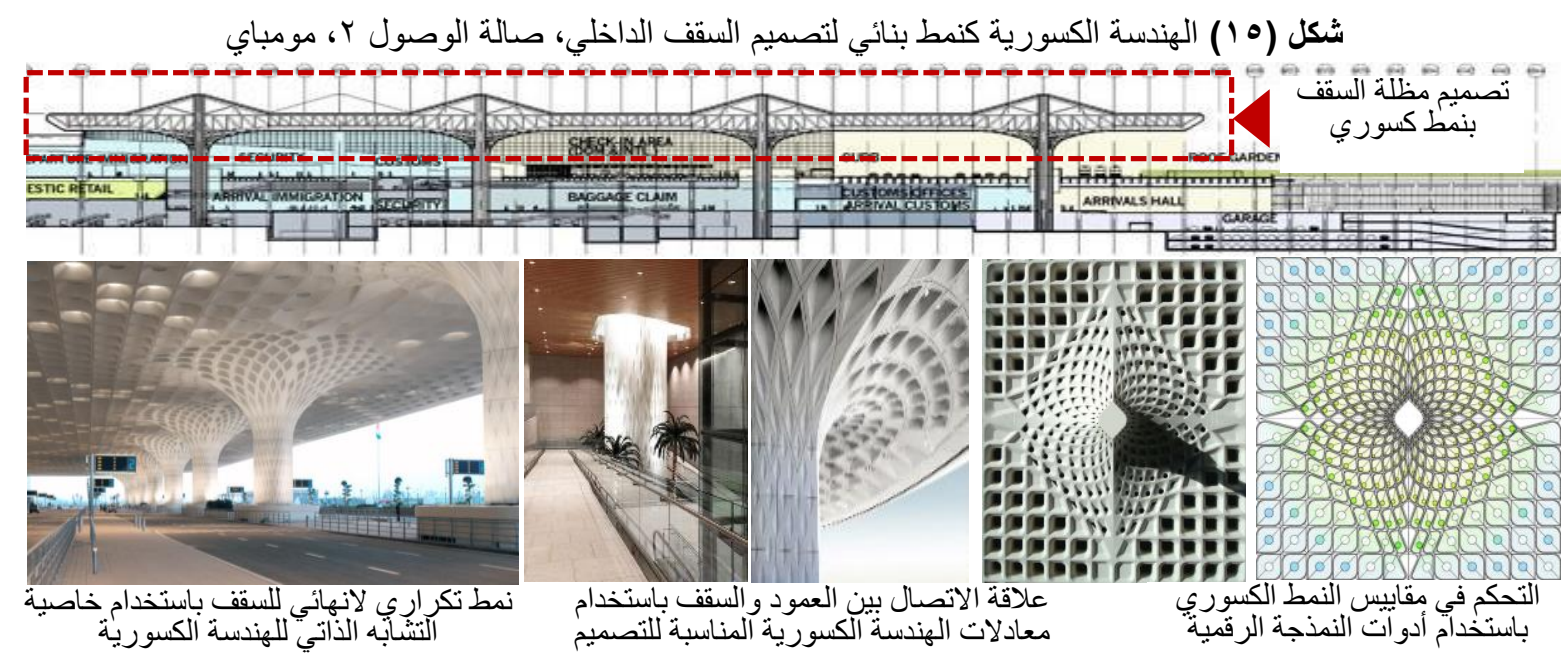

[2014,Designboom ] : المصدر 
• مشروع تغيير واجهة مول ليفربول، المكسيك، تصميم Rojkind Arquitectos، حاصل على جائزة دولية

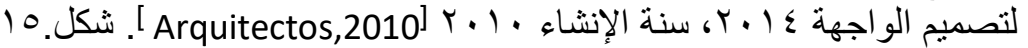

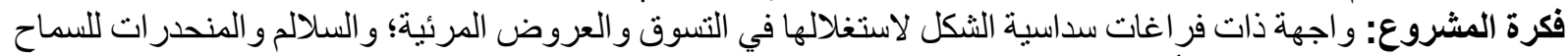

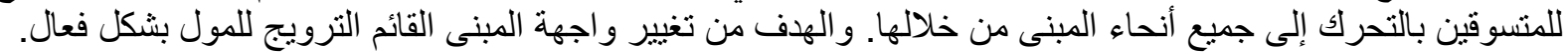

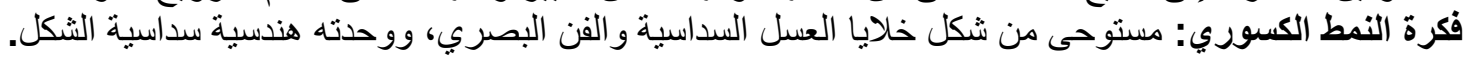
دور النمط وأثره في التصميم: استغلال النمط التصميمي كوحدات متعددة الوظأئف بأحجام مختلفة، ديناميكية الو اجهة.

شكل(7 1 ) الهندسة الكسورية كنمط بنائي لتصميم واجهة فر اغية، مول ليفربول، المكسيك -
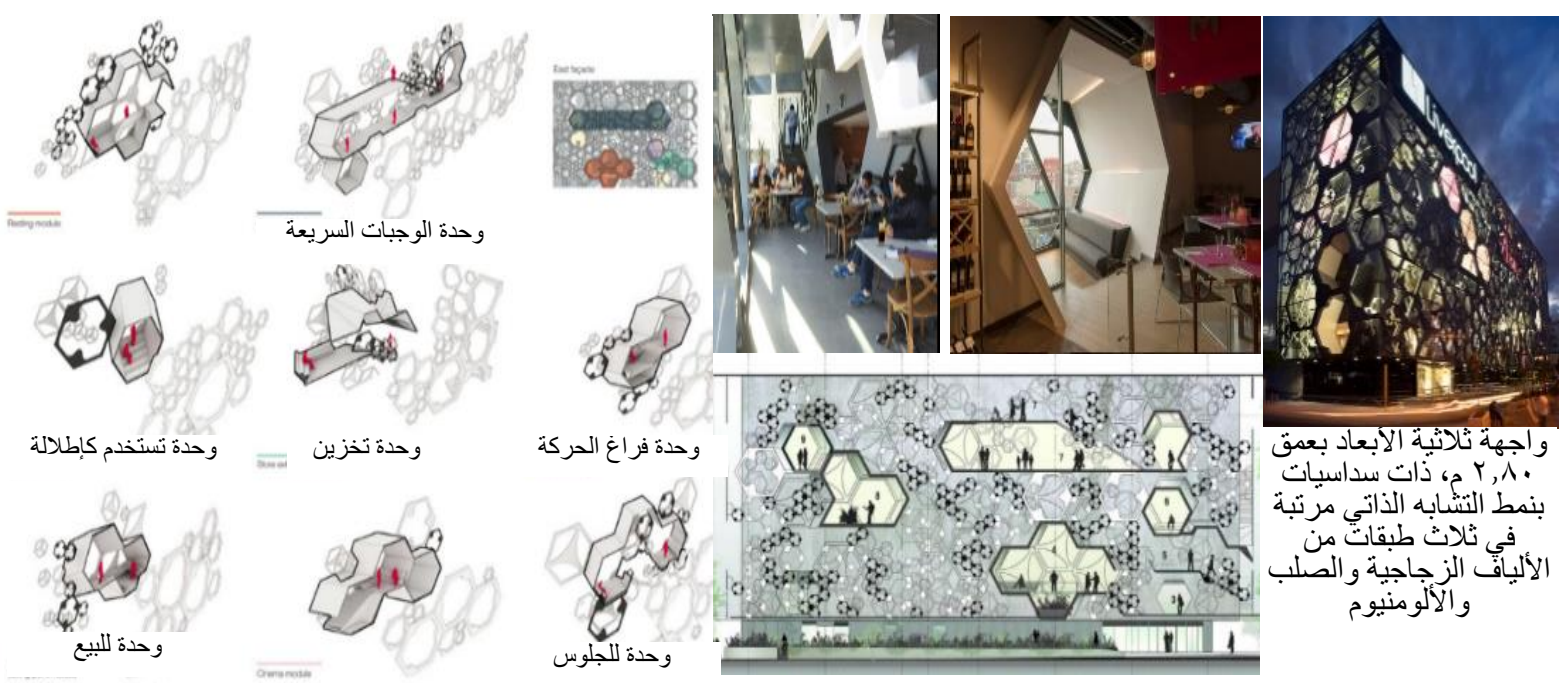

المصدر : [Arquitectos, 2010]

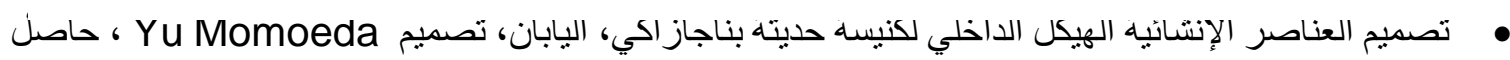

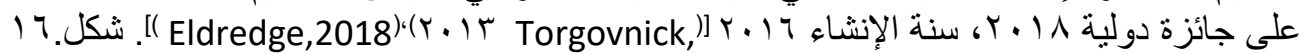

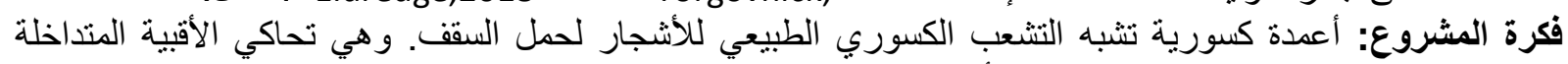

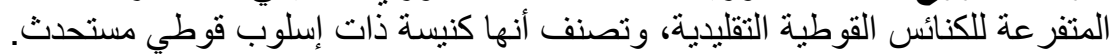

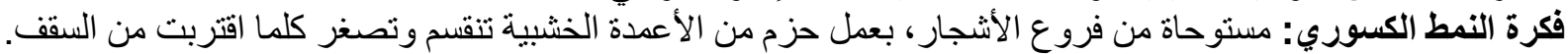

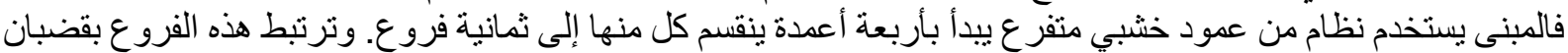
من الصلب الأبيض وتدعم بدور ها المستوى التالي من ثمانية أعمدة أصغر ، تتفرع لاعم الجزء ألعاء العلوي من دور النمط وأثره في التصميم: ربط المبنى بالمحيط الطبيعي، وحمل سقف الكنيسة. هذا النوع من التصميم يوضح مدى الإلى الجمال و الابهار لاستخدام الهندسة الكسورية ثلاثي الأبعاد. شكل (7 1 ) الهنسة الكسورية كنمط بنائي لتصميم العناصر الانثائية كنبسة نجاز اكي، اليابان

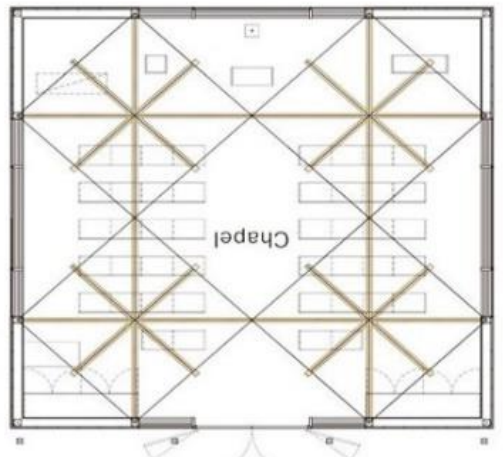

تصميم طبيعةطاطسطفاعية باستخدام النظام الكسوري

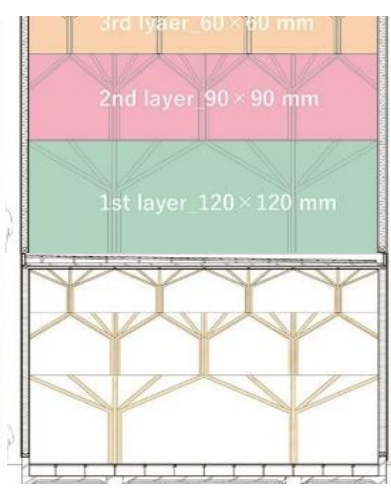

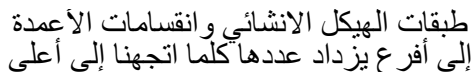
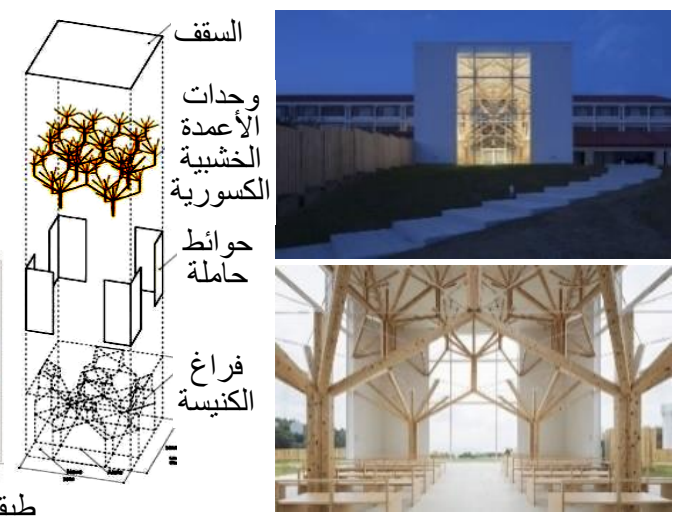

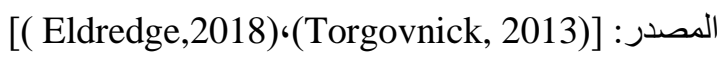

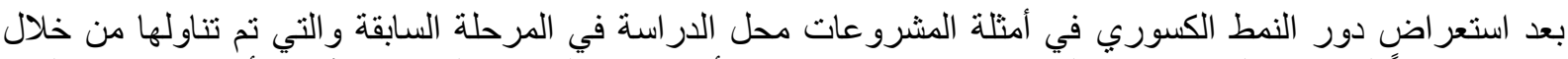
تصنيفها طبقاً لمستوى التصميم في العمارة وعناصره، نستنبط أن مفهوم الهندسة الكسورية يُمكن أن يساهم في الفكر 


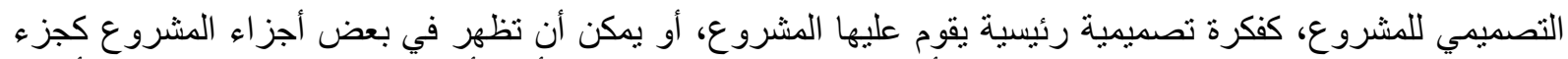

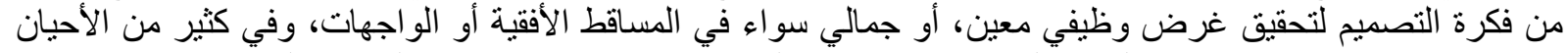

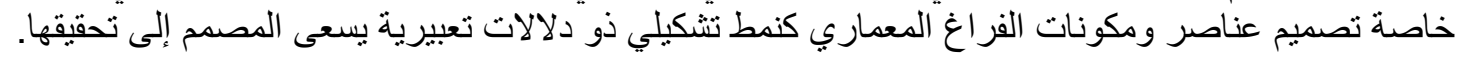

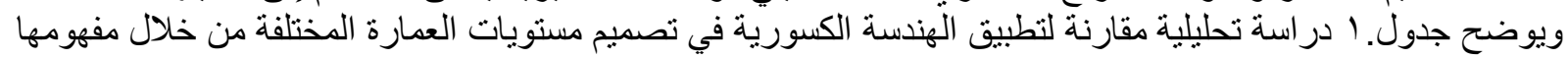

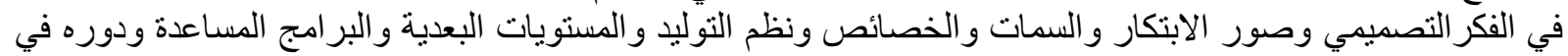
التصميم لمشرو عات محل الدر اسة.

جدول (1) تحليل مقارن بين المشرو عات محل الدر اسة لتطبيق الهندسة الكسورية في مستويات التصميم، المصدر : الباحث

\begin{tabular}{|c|c|c|c|c|c|c|c|c|c|c|c|c|}
\hline \multicolumn{7}{|c|}{ تصمد النتاح المعماءع) معناصه المختلفة } & \multirow{2}{*}{\multicolumn{2}{|c|}{ تنسيق موقع }} & \multirow{2}{*}{\multicolumn{2}{|c|}{ تصميم عمراني }} & \multirow{2}{*}{\multicolumn{2}{|c|}{ مستويات التصميم في العمارة }} \\
\hline عنصر & داخلى & الفرا. & & اصر الكا & & الكتلة & & & & & & \\
\hline 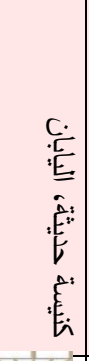 & 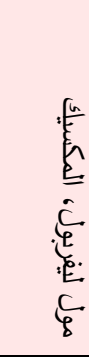 & 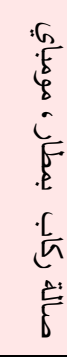 & 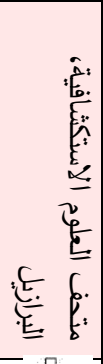 & 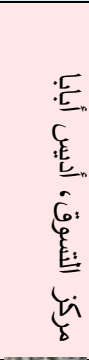 & 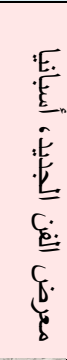 & 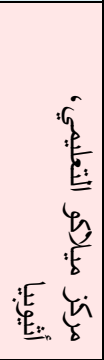 & 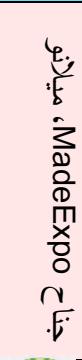 & 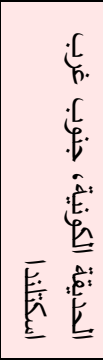 & 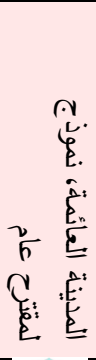 & 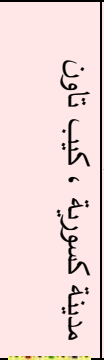 & 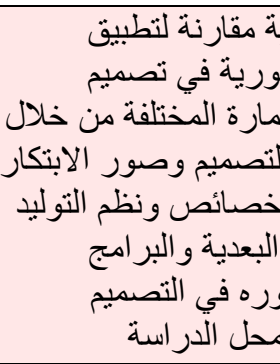 & 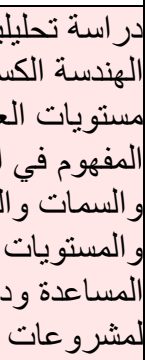 \\
\hline W & 8 & 8 & گૅ & Mas & & 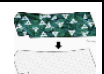 & \& & 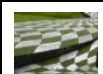 & 8 & E: & لـا الكسوري المستخدمة & وحدة النمد \\
\hline & & & & & & & & & & & فكرة التصميح . & مفهوم \\
\hline & & & & & & & & & & & لرزء من فكرة التصميح & الكسورية في \\
\hline & & & & & & & & & & & |هندس لصميمي سليلمة & صور إتكار \\
\hline & & & & & & & & & & & (2) & 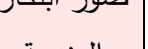 \\
\hline & & & & & & & & & & & هبريغي & الهندسة \\
\hline & & & & & & & & & & & لرنامنكية & \\
\hline & & & & & & & & & & & اتكونات معقدة & : \\
\hline & & & & & & & & & & & أجزاءوها غر متجانسة & $\xi$ \\
\hline & & & & & & & & & & & هياكل ربط بتعدد مستويات & $\underline{\underline{E}}$ \\
\hline & & & & & & & & & & & تاتثابه بصري،انسجام & E. \\
\hline & & & & & & & & & & & لمتنامية لانهائية & 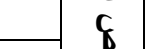 \\
\hline & & & & & & & & & & & الثشابه ذاتع، & $\sum_{n}^{n}$ \\
\hline & & & & & & & & & & & أبعاد كسورية & E. \\
\hline & & & & & & & & & & & ال التكون عبر التكرار & {[} \\
\hline & & & & & & & & & & & الكسورية العشوائية & E: \\
\hline & & & & & & & & & & & إلتترج الحجمي. & \\
\hline & & & & & & & & & & & إل الثيوادى، والمولدات & \\
\hline & & & & & & & & & & & | الدوال التكرارية IFS & E \\
\hline & & & & & & & & & & & L-system & \\
\hline & & & & & & & & & & & ثلثائــ الأسعاد & أبعاد النمط \\
\hline & & & & & & & & & & & لـلاثي, الأبعاد & \\
\hline & & & & & & & & & & & إحراسيهوبر & برامج رقمية \\
\hline & & & & & & & & & & & 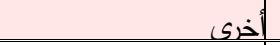 & كساعدة \\
\hline & & & & & & & & & & & هظظف & \\
\hline & & & & & & & & & & & إنشائع, & E. \\
\hline & & & & & & & & & & & ليئح. & 6 \\
\hline & & & & & & & & & & & إجمالد, - la & \\
\hline & & & & & & & & & & & لمسقط أفق & 5 \\
\hline & & & & & & & & & & & لمساقط رأسية & 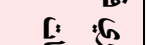 \\
\hline & & & & & & & & & & & اجية اجهة & $\xi$ \\
\hline & & & & & & & & & & & | ليقف & $\underline{E}$ \\
\hline & & & & & & & & & & & أغلاف المين & E \\
\hline & & & & & & & & & & & فتحات & $x_{0}$ \\
\hline & & & & & & & & & & & |عناصر انشائية & Ei: \\
\hline
\end{tabular}




\section{ه تفسير نتائج التطليل المقارن للمشاريع محل الدراسة}

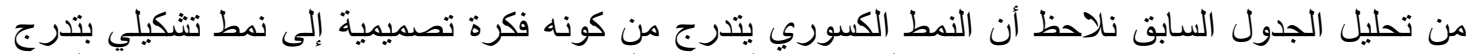

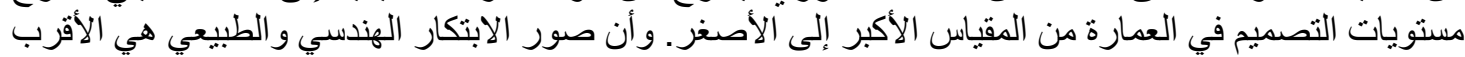

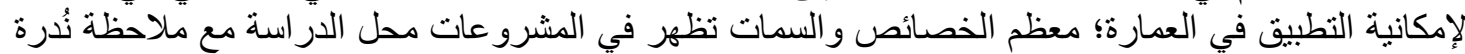
خاصية الكسور العشوائية لأنها بالغة التعقيد وتحتاج عناصر بمسطحات كبيرة، وقدرات خاصنة في التفكير الابداعي.

يتنوع النمط الكسوري بين التقليدي أو الابتكاري أو الأكثر تعقيداً؛ بالر غم من أنه في جميع الحالات يعتمد

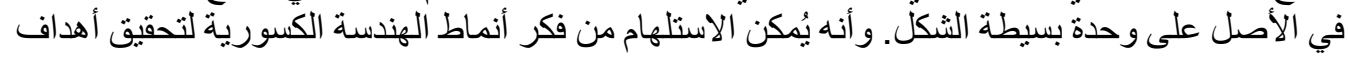

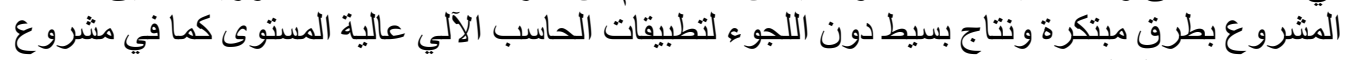
مركز ميلاكو التعليمي.

يندر جدور النمط الكسوري في المشروعات محل الدر اسة تنتازلياً بداية من الجمالي وتحقيق القيم الخاصة

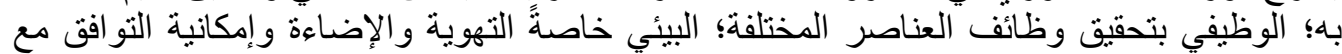
الأنشطة المختلفة؛ الإنشائي نتيجة خصائصائ الطناص وسماته التشكيلية.

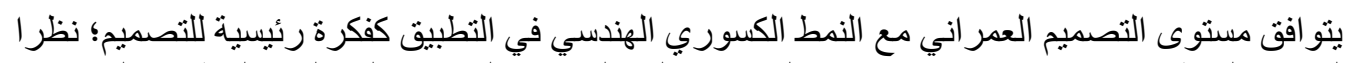

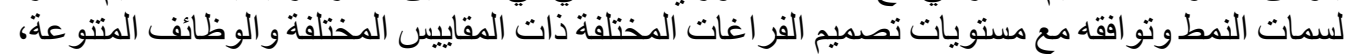

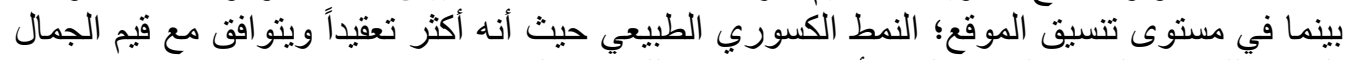
الحسية للطبيعة الحية والسياق العام بأكثر من مفهوم للهندسة الكسورية.

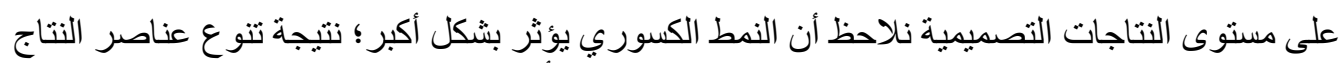

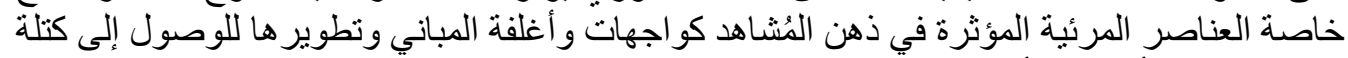

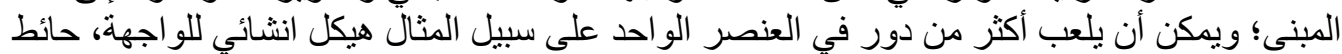

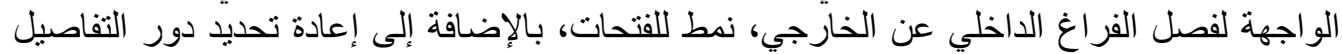

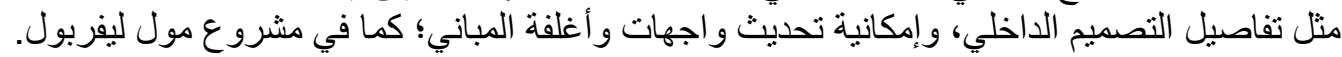

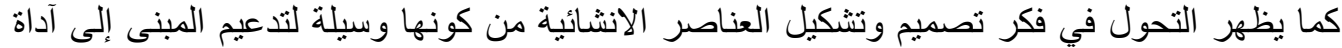

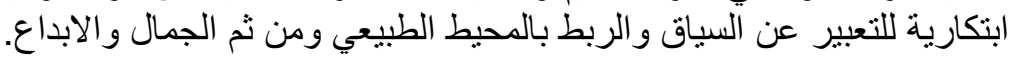

أنه يُمكن صياغة دور نمط الهندسة الكسورية في تحقيق الوظيفة في العمارة كما يلي:

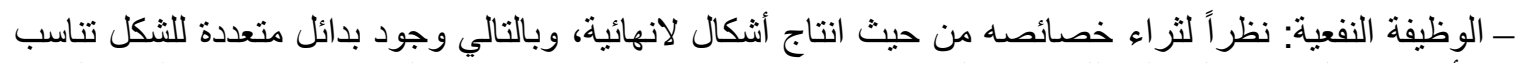

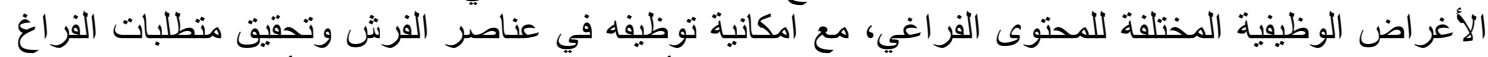

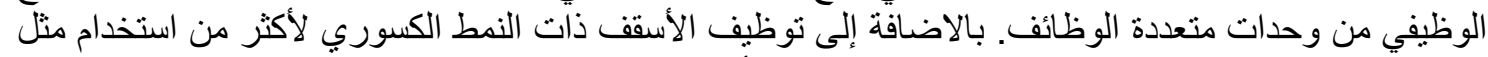
وضع وحدات الاضاء الصناعية دون الحاجة لاستخدام الأسقف الاستعارية.

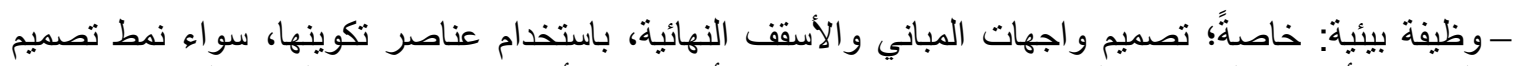

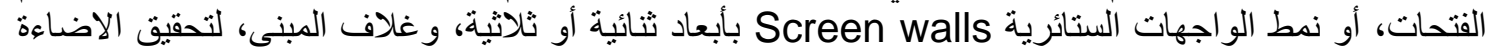

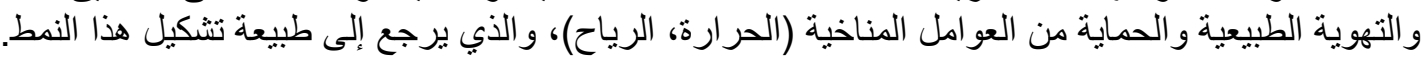

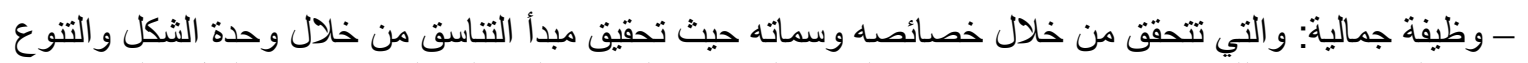

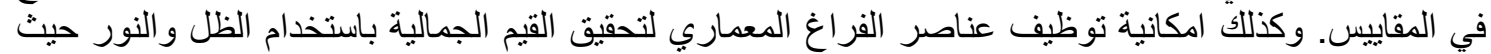
البعد الفر اغي للنمط، وتوظيف كالعناصر الانشائية ذات التنكيل الكسوري لتحقيق لتهيق الوظيفة الجمالية.

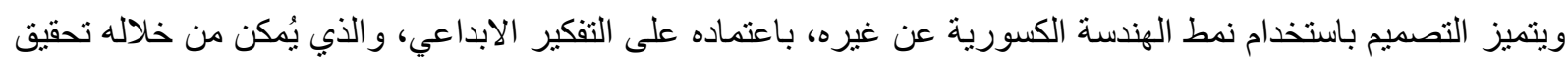

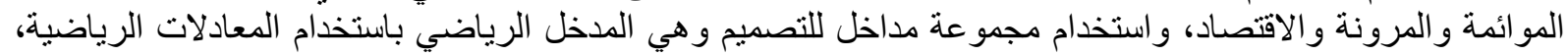

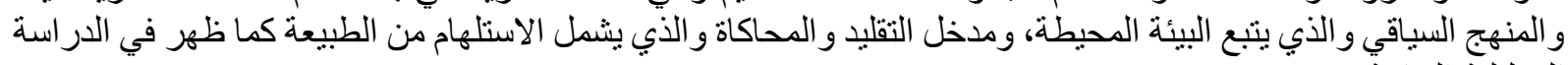

التحليلية السابقة.

ويمكن إجمال تفسير النتائج التي توصلت إليها الدر اسة، و استخلاص الدروس المستفادة في شكل إطار فكري عام لتأثير دور

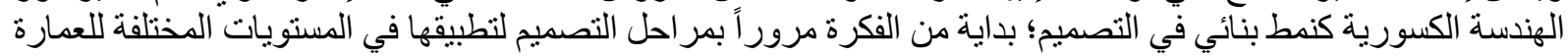

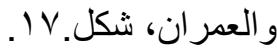


شكل (IV) إطار فكري عام لتأثير دور الهندة الكسورية كنمط بنائي للتصميم في المستويات المختلفة للعمارة

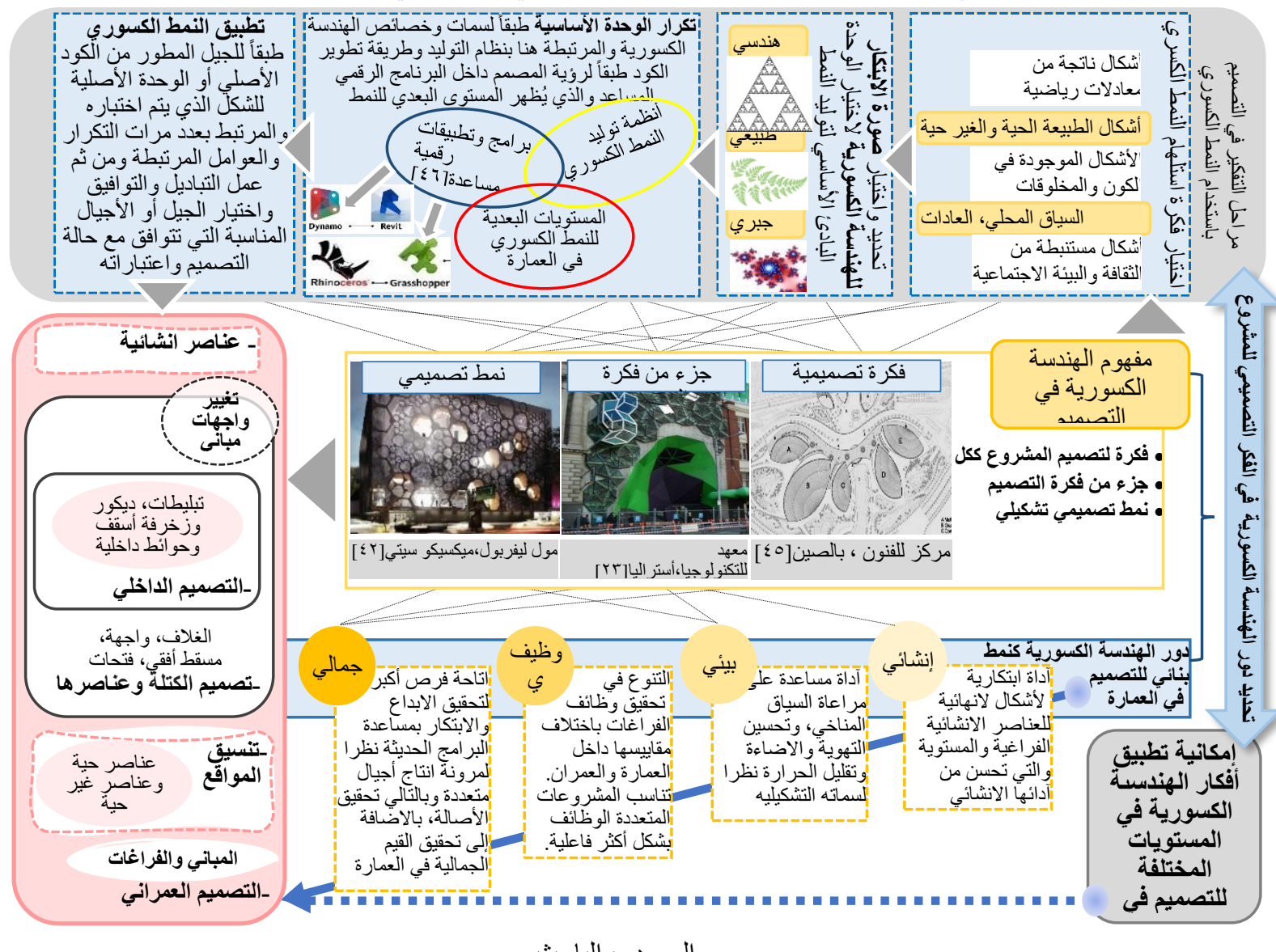

المصدر : الباحث

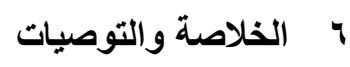

1/7 الخلاصة

• تُعد الهندسة الكسورية من أفضل الخيار ات لتمثيل النوع الجديد من نظام النموذج؛ قادر على المرونة في بناء نظام

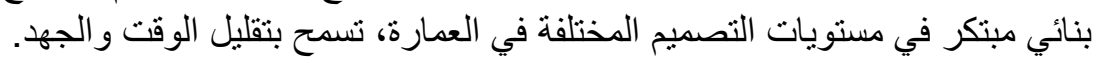

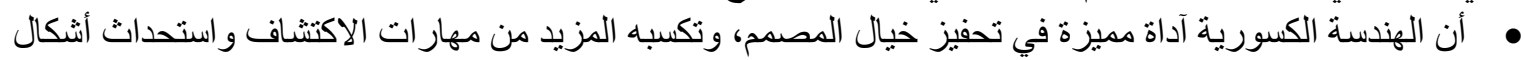

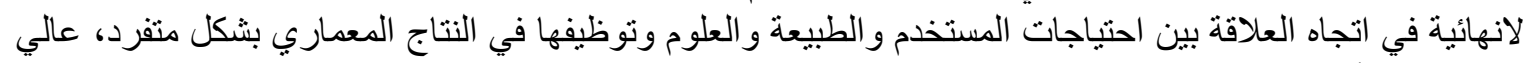

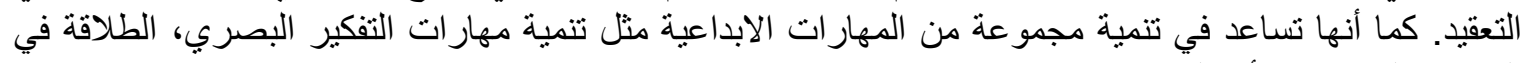
التفكير و المرونة و الأصالة.

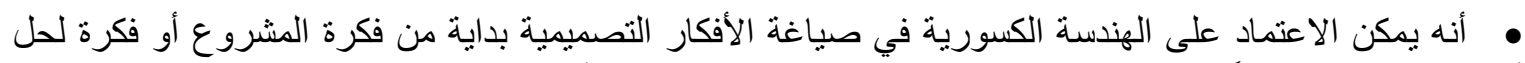

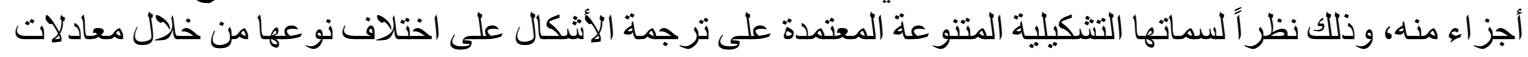

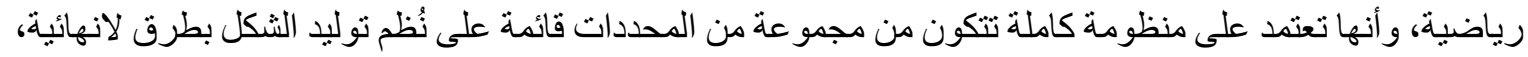

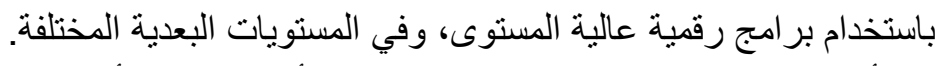

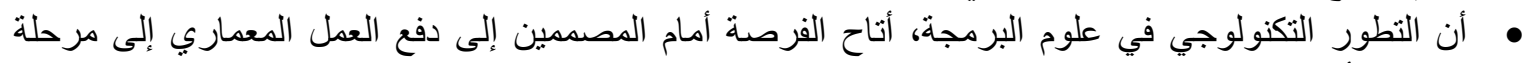

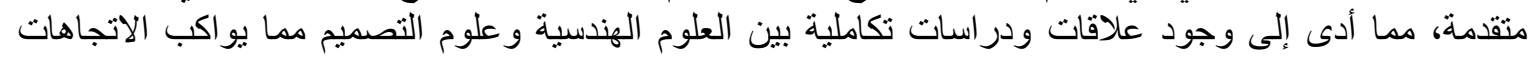

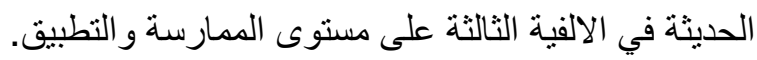

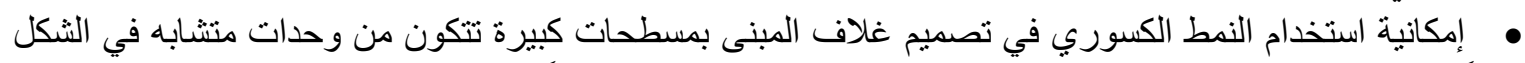

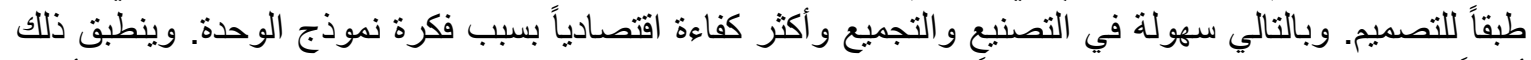

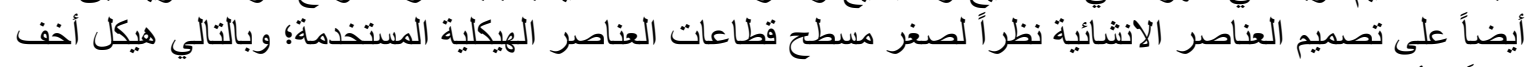
وزناً، و أقلى تكلفة. 
T/ץ التوصيات

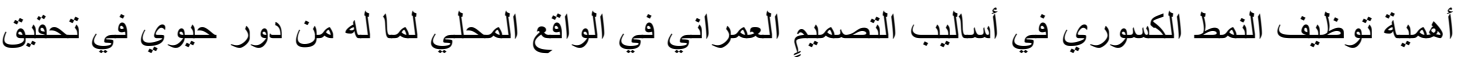

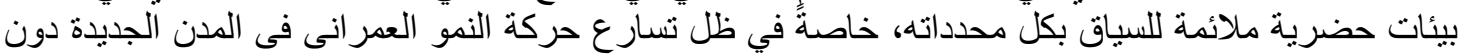

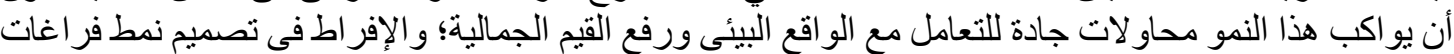

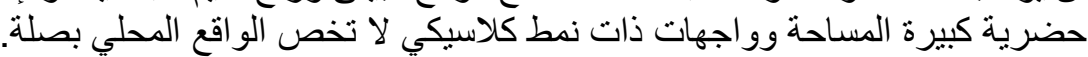

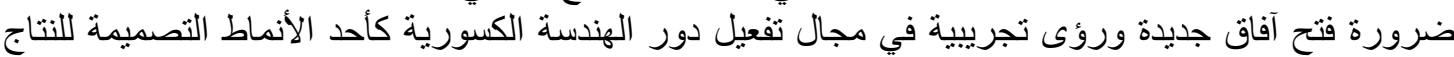

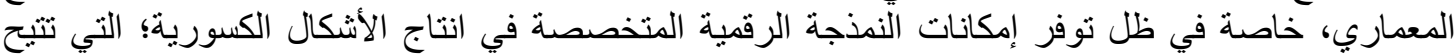
الفرصة للمعماريين لخلق تصميمات متفردة، مما بساهم في الارتقاء بقيمة المنتج المعماري وتأثنيره الإيجابي في التي

المحيط.

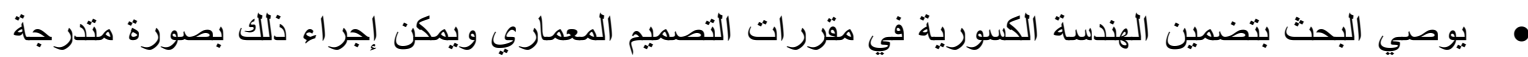

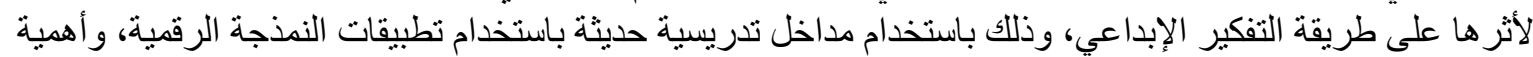

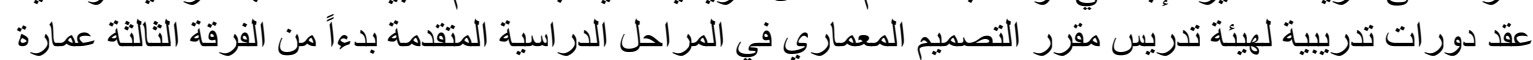

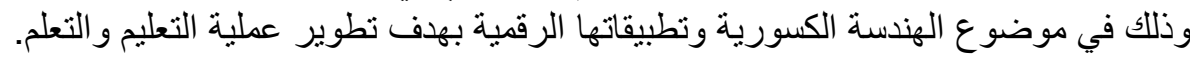

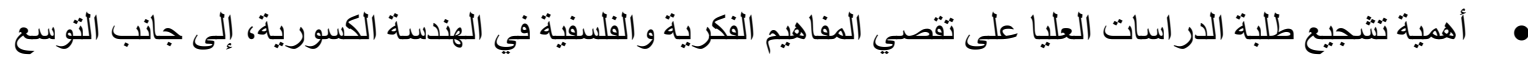

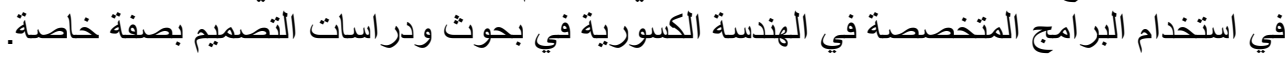

\section{References}

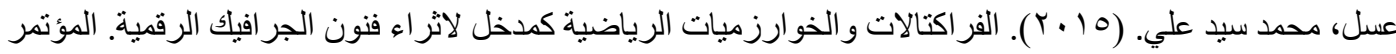

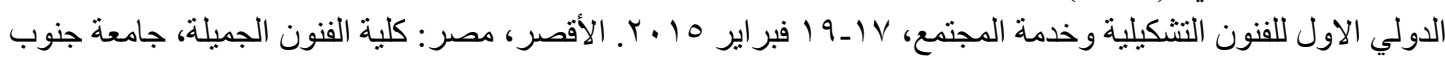
الو ادي.

Assal, M. S. (2015). Fractal and Algorithm Arts as input to enrich the Digital Graphic art. First International Conference of Plastic Arts and Community Service, 17-19 February 2015. Luxor,

Egypt: South Valley University.

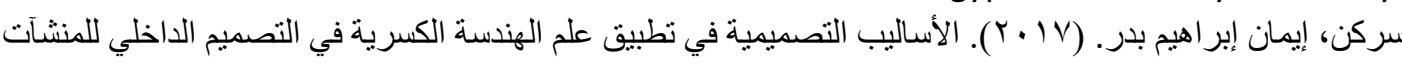

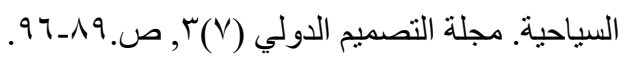

Sarkan, I. (2017). Design methods in the application of fractal geometry in the interior design of tourist buildings. International Design Journal, 7 (3), 89-96.

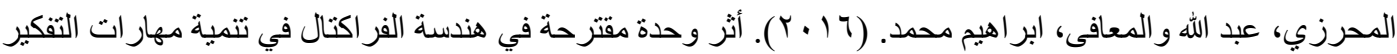

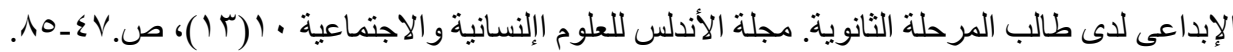

Al-Mahrezi, A.A. and Al-Moafe, I.M. (2016): The Effect of Fractals Geometry on Improving Creative Skills of Thinking of Secondary Grade Students. Al-Andalus journal for Humanities \& Social Sciences.10 (13), 47-85.

ArchDaily (2009). Melaku Center / xvstudio. Retrieved from: https://www.archdaily.com > ArchDaily , Articles > Melaku Center / xvstudio/ acssesed 9/4/2019.

ArchDaily (2016). Lideta Market / Vilalta Studio. Retrieved from: https://www.archdaily.com/789535/lideta-market-vilalta-arquitectura/ acssesed 11/3/2019

Archdaily (2018). The Fuzhou Strait Culture and Art Centre / PES-Architects. Retrieved from: https://www.archdaily.com/904225/the-fuzhou-strait-culture-and-art-centre-pes-architects/ accessed at 10/7/2019.

Architecture, Fractal \& Nature (2013). Posts tagged 'Nature'. Retrieved from: https://iasefmdrian.wordpress.com/tag/nature/ acssesed at 1/5/2019.

Architecture, Fractal \& Nature (2013). Posts tagged 'Nature'. Retrieved from: https://iasefmdrian.wordpress.com/tag/nature/ acssesed 9/6/2019. 
Arquitectos, Rojkind. (2010). https://www.archdaily.com/560641/liverpool-insurgentes-departmentstore-rojkind-arquitectos/ acssesed 13/5/2019

Arts, Mathematics, and Ardlitecture, Pp.235-240.

https://archive.bridgesmathart.org/2003/bridges2003-235.pdf acssesed at 6/4/2019.

Belma \& Ayyildiz, Alik ,Sonay.(2016) "Fractals and Fractal Design in Architecture", 13th International Conference "Standarization, Protypes and Quality: A Means of Balkan Countres' Collaboration", Romania, November, p.284,285.

Britton, Brian. (2012). https://www.behance.net/gallery/7805665/Thesis-2012-Island-City / acssesed $7 / 6 / 2019$.

Byng, Malaika (2009). Exploratory Science Museum by CHN Arquitetos. Retrieved from: https://www.dezeen.com/2009/09/07/exploratory-science-museum-by-chn-arquitetos/ acssesed 13/5/2019

Carpo, Mario. (2013). "The Digital Turn in Architecture 1992-2012", John Wiley \& Sons Ltd, p.99.

COHN, DAVID. (2013). https://www.architectural-review.com/today/contemporary-artcentre-spain-by-nieto-sobejano acssesed 19/4/2019

Designboom (2014). SOM unites mumbai airport terminal with fractal roof canopy. Retrieved from: http://www.designboom.com/architecture/som-unites-mumbai-airport-terminal-with-fractalroof-canopy-02-25-2014/ acssesed 13/5/2019

Designboom (2017). VILALTA completes mall in ethiopia with perforated envelope. Retrieved from: https://www.designboom.com/architecture/vilalta-arquitectura-lideta-mercato-shoppingmall-addis-ababa-ethiopia-12-26-2016/ acssesed 15/4/2019

Dutta,Tanisha.(2018) "Shapes, Patterns and Meanings in Indian Temple Architecture", American Journal of Civil Engineering \& Arch., Vol. 6, Pp.206- 215.

Edward, Louis . (2006). http://www.evolo.us/fractal-city-in-cape-town-urbanproject/acssesed1/5/2019.

Efe, Recep. (2016). "Environmental Sustainability and Landscape Management", St. Kliment Ohridski University Press Sofia, P. 188-202.

Eldredge, Barbara (2018). Stunning Japanese chapel showcases tree-like fractal columns, The chapel's interior structure is made of stacked timber pillars that split and shrink as they ascend to the ceiling. Retrieved from: https://www.curbed.com/2018/1/4/16848894/japan-chapelarchitecture-fractal-yu-momoeda accessed at 10/7/2018

Flake, Gary. (2000). "The Computational Beauty of Nature: Computer Explorations of Fractals, Chaos, Complex Systems and Adaptation", Cambridge, The MIT Press.

Harris, James. (2012) "Fractal Architecture: Organic Design Philosophy in Theory and Practice", 2012.

http://fedsquare.com/about/history-design/ acssesed at 16/4/2019.

https://www.researchgate.net/publication/325020334_The_Garden_of_Cosmic_Speculation_The_M ystery_that_is_Nature_a_dialogue_with_the_cosmos/ acssesed 9/6/2019.

Jencks, Charles. (2011) "The Universe in the Landscape", https://www.tandfonline.com/doi/full/10.1080/14688417.2012.753332/ .

Kitchley, "Jinu. (2003). FRACTALS IN ARCHITECTURE", January 2003, https://www.researchgate.net/publication/256057394_Fractals_in_architecture/ acssesed at 16/4/2019.

majalinse, (2016). Fractal geometry in nature and architecture, https://spatialexperiments.wordpress.com/2016/09/18/fractal-geometry-in-nature-andarchitecture/. 
Mandelbrot, Benoit B. (1983). "The Fractal Geometry of Nature", New York, W. H. Freeman and Company.

Mirmoradi, Seyedeh. (2017). "Recognition of the role of nature in the formation of fractal architecture", Technology and Management in Construction, Pp.1576,1577-1581.

Nicoletta, Sala (2006) "Fractal geometry and architecture: some interesting connections", EcoArchitecture: Harmonisation between Architecture and Nature, Vol 86, WIT Press, p.163,164,167.

NRES (2008). FRACTAL APPLICATIONS IN LANDSCAPE ECOLOGY. Retrieved from: http://www.johnboccio.com/courses/CFSCE_2018/landscape.pdf acssesed at 11/3/2019.

Osama, Amal. (2014) "Fractal geometry in architecture: from formative idea to superficial skin design", ARCHDESIGN'14, p.42

Rian, Mario Sassone and Shuichi Asayama\& lasef. (2018). "From fractal geometry to architecture: Designing a grid-shell-like structure using the Takagi-Landsberg surface", Computer-Aided Design, Vol.98, Pp. 40-43.

Riana \& Chang, lasef Md, Jin-Ho Parka, Hyung Uk Ahna, Dongkuk. (2007). "Fractal geometry as the synthesis of Hindu cosmology in Kandariya Mahadev temple", Building and Environment, Vol. 42, p. 4093-4107.

Rumiez, Agnieszka. (2014). "Fractal Architecture”, Scientific Journal of Riga Technical University Architecture and Urban Planning-, Vol. 8, p.47.

Saad, Rania. (2016). "Furniture design inspired from fractals", International Design Journal, Vol. 6, Pp. 170-175.

Sala, Nicoletta. (2013). "Fractal Geometry and Self-Similarity, In Architecture: An Overview Across the Centuries", ISAMA, The International Society of the Arts, Mathemattics, and Architecture, Pp.235-240.

Shiffman, Daniel. (2012). THE NATURE OF CODE, https://natureofcode.com/book/chapter-8-fractals/ acssesed at 29/5/2019.

Szalapaj, Peter. (2005). "Contemporary Architecture and the Digital Design Process", Elsevier, Delhi, p.48.

Torgovnick, K. (2013). Architecture infused with fractals: how TED speaker Ron Eglash inspired architect Xavier Vilalta. Retrived from: https://blog.ted.com/architecture-infused-with-fractals-roneglash-and-xavier-vilalta/ accessed at 10/7/2018

Wintour, Paul. (2016). Fractals \& L-Systems https://parametricmonkey.com/2016/03/09/fractals-2/ acssesed at 2/6/2019.

Wintour, Paul.(2016). Fractals \& L-Systems https://parametricmonkey.com/2016/03/09/fractals-2/ acssesed at 2/7/2019.

Wordpress.com (2019). Retrieved from: https://arch505a.files.wordpress.com/.../t3-struconcretecontemporary-arts-center-cc3b/ acssesed 19/5/2019

Zappulla, Carmelo. (2013). "Connections Between Architectural Design and Mathematical Patterns", p. 80,82 .

https://www.academia.edu/5309062/Connections_Between_Architectural_Design_and_Math ematical_Patterns/ acssesed at 11/3/2019. 


\title{
Fractal Geometry as a Structural Pattern for Design in Architecture
}

\section{Mona Mohamed Hosni}

Department of Architecture, Faculty of Engineering, El Materiya, Helwan University

\begin{abstract}
The digital boom and computer applications used in architecture especially 3D applications and programs have influenced the thought and design methods of architecture, leading to the emergence of new vocabulary and different architectural languages that are more sophisticated and liberal, characterized by a great deal of diversity, dynamism, and complexity that are difficult to emerge through traditional design methods. The research discusses one of the new architectural applications of parametric design of fractal geometry to explore the possibilities of application in architecture.

The research raises the following question: What are the possibilities of applying fractal geometry at different levels of design in architecture? Is there a relationship between the use of the fractal pattern and the achievement of space functions, environmental solutions, and other aspects of design and considerations?
\end{abstract}

The research deals with the study of the concept of fractal geometry, with a trace of its historical development; images of its innovation, features and characteristics; and then study methods and tools to generate a fractal pattern and analysis of the possibilities of application at different levels of design. This to create a basis of knowledge that helps the designer and researcher in this language of programming and determine the possibilities of activating this pattern in the design and its determinants to develop innovative design products to suit the ocean, and to show lessons learned and return to society.

The research concludes with findings and recommendations, the most important is that fractal geometry is one of the best options for formulating innovative design ideas. The research recommends the importance of employing fractional geometry in architectural and architectural design methods, with the need to include them in architectural academic studies and push towards the importance of practicing specialized modeling programs to enable the translation of complex ideas and saving time and effort.

Keywords: Fractal, Fractal Geometry, Fractal Design, Self-Similarity, Algorithms. 